\title{
Salaires et Emploi dans les Secteurs Public et Privé, Différences et Interactions
}

\author{
Jake Bradley, University of Cambridge \\ Matt Dickson, University of Bath \\ Fabien Postel-Vinay, University College London \\ Hélène Turon, University of Bristol ${ }^{1}$
}

\section{Mai 2016}

\section{Introduction}

La masse salariale du secteur public représente environ un cinquième de la dépense publique dans la plupart des pays Européens. A une époque où les économies budgétaires sont régulièrement en première ligne du débat politique, il nous paraît important de bien comprendre si les conditions de rémunération et d'emploi offertes dans le secteur public sont avantageuses par rapport à celles du secteur privé, ainsi que d'évaluer l'intensité de la compétition entre les deux secteurs pour l'offre de travail, pour être en mesure de prédire l'impact potentiel de différents choix politiques en termes d'emploi et de salaires dans le secteur public. Nos travaux (Postel-Vinay et Turon, 2007, Dickson, Postel-Vinay et Turon, 2014, et Bradley, Postel-Vinay et Turon, 2015) apportent des éléments de réponse à ces questions.

Nous allons dans un premier temps documenter les différences intersectorielles d'emploi et de salaires dans six pays Européens pendant la période 1994-2001 à l'aide de la base de données fournie par le European Community Household Panel. Ces pays sont l'Allemagne, I'Espagne, la France, I'Italie, les Pays-Bas et le Royaume-Uni. Notre estimation des différences de salaires en coupe ainsi que des différentes dimensions dynamiques des processus de salaires dans les deux secteurs nous permet d'évaluer de primes de longterme du secteur public. Celles-ci représentent les différences de valeurs à long terme de l'emploi dans les deux secteurs qui incorporent, pour des agents à anticipations rationnelles, non seulement les différences de salaires en coupe mais aussi les différences de perspectives en termes de progression et volatilité des salaires futurs et de perte d'emploi. Notre approche empirique innovante nous permet de tenir compte des effets de sélection des employés dans le secteur public et d'estimer des trajectoires dynamiques

\footnotetext{
${ }^{1}$ Auteur de correspondance: helene.turon-lacarrieu@bristol.ac.uk. Nos remerciements à Etienne Lalé pour sa relecture attentive.
} 
propres à chaque secteur, et dans une certaine mesure, à chaque individu. Sans établir de causalité, nous mettons nos résultats en perspective en les reliant au cadre institutionnel qui régit l'emploi et les salaires du secteur public dans chaque pays de notre échantillon. Nos résultats montrent que, lorsque les effets de composition sont pris en compte ainsi que les différences entre les secteurs en termes de dynamiques de salaires et d'emploi, la valeur actualisée d'un emploi dans le secteur public en France offre une prime de 6\% par rapport à un emploi dans le secteur privé.

Dans un second temps, nous proposons un modèle structurel permettant de quantifier une réponse de l'ensemble du marché du travail à différentes alternatives de choix politiques en termes d'emploi et de salaires dans le secteur public. Notre modèle vise à reproduire des mécanismes plausibles de concurrence entre les deux secteurs d'emploi et la réaction du secteur privé à un changement de politique du secteur public en termes d'emploi et de salaires. Cette approche structurelle nous paraît être un pendant nécessaire de l'approche descriptive adoptée dans nos travaux précédents. En effet, elle permet de prédire les réactions des différents agents (travailleurs, entrepreneurs du secteur privé) aux changements de politiques contrefactuelles que nous voulons évaluer avant leur éventuelle mise en vigueur.

Nous utilisons l'idée de valeur à long-terme de l'emploi dans chaque secteur introduite cidessus comme vecteur de compétition entre les deux secteurs pour l'offre de travail. En construisant un modèle structurel où les travailleurs peuvent passer du chômage à l'emploi dans un secteur donne, ou changer d'emploi au sein d'un même secteur ou vers un secteur différent sur la base de comparaisons de valeurs à long-terme, nous illustrons la dimension compétitive de l'interaction entre les deux secteurs. Dans notre modèle, pour un choix politique donné en termes de niveaux d'emploi et de salaires dans le secteur public, les entreprises privées décident de manière optimale de leurs niveaux de recrutement et de salaires selon leur niveau de productivité. L'effort de recrutement agrégé et la distribution d'offres de salaires dans le secteur privé sont ainsi modélisés de manière endogène et réagissent à des changement de politiques d'emploi et de salaires dans le secteur public. Ce travail nous permet ainsi d'évaluer et de comparer l'impact potentiel de différentes politiques visant a réduire la masse salariale du secteur public.

Le reste de cet article est organise comme suit: dans la section 2, nous passons en revue les travaux qui nous paraissent les plus proches du nôtre et les plus pertinents pour les questions soulevées ici. Les Sections 3 et 4 présentent nos résultats sur les différences statiques et dynamiques, respectivement, entre les deux secteurs d'emploi. L'agrégation de ces résultats sous la forme de prime de long terme dans le secteur publique est présentée dans la Section 5 . Les phénomènes de sélection non-aléatoires sont discutés dans la Section 6. La section 7 introduit notre modèle structurel, que nous utilisons ensuite dans la Section 8 pour simuler l'impact de différentes politiques possibles de réduction de la masse salariale. La Section 9 passe en revue le cadre institutionnel régissant l'emploi et 
les salaires dans les six pays Européens de notre échantillon. Enfin, la conclusion se trouve dans la Section 10.

\section{Littérature}

La grande majorité de la littérature sur les différences salariales entre public et privé évalue les différences salariales dites statiques: I'attention est portée sur les différences de salaires a un instant précis, estimées à l'aide de données en coupe. Dans toutes ces études (par exemple Disney et Gosling, 2003, pour le Royaume Uni, Dustmann et van Soest, 1998 et Melly, 2005, pour l'Allemagne, Hartog et Oosterbeek, 1993, et van Ophem, 1993, pour les Pays-Bas, Bargain et Melly, 2008, pour la France et Lassibille, 1998, pour l'Espagne), une large part de ces différences viennent des effets de sélection entre secteurs, la composition des populations de travailleurs dans les deux secteurs présentant des différences en termes d'éducation, d'occupation et de genre.

Pour la France et l'Italie, Lucifora et Meurs (2006) concluent que la majorité des différences de salaires entre les deux secteurs vient du fait que les salaires sont déterminés par des accords collectifs dans le secteur privé, et que l'expérience et l'éducation y jouent un rôle plus important du fait du pouvoir de négociation des syndicats. Leurs résultats de régression par quantiles s'accordent avec les résultats de Melly (2005) pour l'Allemagne et montrent que la proportion des différences salariales intersectorielles qui peut s'expliquer par des différences de caractéristiques observées croît lorsqu'on grâvit l'échelle des quantiles de la distribution des salaires. Le travail de Ghinetti et Lucifora (2007) avec la vague 2001 du panel ECHP confirme cette conclusion.

Cependant, même si ces travaux sont informatifs s'agissant des différences entre secteurs, et si certains tiennent compte de l'endogénéité du choix de secteur soit à l'aide d'une hypothèse sur la forme fonctionnelle de cette sélection (van Ophem, 1993) soit à l'aide d'une variable instrumentale (Dustmann et van Soest, 1998, Hartog et Oosterbeek, 1993), ils n'estiment que des différences instantanées de de salaires entre secteurs et ignorent tout aspect dynamique de ces différences.

Cappellari (2002) est à notre connaissance la seule autre étude qui s'intéresse également aux différences de trajectoires dynamiques des salaires entre les secteurs public et prive. II utilise un panel de données administratives Italiennes et fait I'hypothèse (forte) d'une sélection exogène entre les secteurs.

Notre contribution à cette littérature est double : de manière descriptive puis structurelle, nous évaluons des réformes potentielles du secteur public dans un contexte de réduction budgétaire. Nos travaux sur le Royaume Uni (Postel-Vinay et Turon, 2007) et sur cinq 
autres pays Européens (Dickson et al. 2014) apportent une dimension dynamique à la mesure des différences salariales entre privé et public. Comme nous allons le montrer, les différences statiques entre les niveaux de salaires instantanés sont certes présentes dans les données, mais elles ne révèlent qu'une image incomplète de la valeur d'un emploi dans chaque secteur dès lors que des différences importantes en termes de volatilité et de croissance des salaires ainsi qu'en termes de sécurité de l'emploi existent. Notre mesure de valeur de long terme, inspirée de la littérature sur les inégalités de revenus (Buchinsky et Hunt, 1999, Bonhomme et Robin, 2009, Flinn, 2002, et Bowlus et Robin, 2004), incorpore toutes ces dimensions pertinentes dans une valeur de long terme de l'emploi dans chaque secteur. D'autre part, nous tenons compte de la sélection endogène des travailleurs dans les deux secteurs et offrons un complément 'dynamique' à la littérature qui adopte cette démarche pour évaluer les différences sectorielles statiques.

L'approche sous forme réduite des différences entre secteurs peut certes être utile pour illustrer l'importance des différences existantes, ainsi que les aspects statique et dynamique de ces différences. Cependant, elle ne nous renseigne pas sur les mécanismes plausibles pouvant donner lieu à ces différences. Elle ne nous permet pas non plus de prévoir l'impact sur le marche du travail global -- c'est-à-dire comprenant les deux secteurs -- d'un changement de politique publique en termes de salaires et d'emploi dans le secteur public. Une approche structurelle, en revanche, peut satisfaire à cet objectif ; celle-ci fait l'objet de notre troisième travail sur ces différences (Bradley et al. 2015).

Etant données l'importance du secteur public sur le marche du travail (entre un cinquième et un quart de l'emploi total dans le plus grand nombre de pays de l'OCDE) et la fréquence des débats politiques et publics sur le niveau des salaires dans l'emploi public, il est surprenant que si peu de travaux aient été effectués pour modéliser l'interaction entre les deux secteurs. Des contributions récentes (Algan et al., 2002, Quadrini et Trigari, 2007, Horner et al., 2007, Gomes, 2015) analysent l'impact du secteur public sur le niveau et la volatilité des salaires de tous les employés et de l'emploi global. Ces approches s'appuient sur un modèle de recherche dirigée dans laquelle les travailleurs concentrent leur recherche d'emploi dans un secteur particulier (public ou privé) ; elles ignorent la réallocation directe d'emploi à emploi (intra- ou inter-secteur) ; elles font l'hypothèse que les salaires résultent d'une négociation sur le partage du surplus dans l'appariement de la paire firme-travailleur.

Algan et al. (2002) prédisent que la création d'emplois publics donne lieu à un effet d'éviction considérable, si bien que l'emploi public marginal détruirait jusqu'à 1.5 emplois dans le secteur privé dans certains pays de I'OCDE. Cet effet d'éviction est accentué lorsque les salaires publics sont élevés et/ou lorsque les biens et services produits par les deux secteurs sont de proches substituts. Quadrini et Trigari (2007) s'intéressent à la cyclicalité de l'emploi et simulent deux scenarios dans lesquels les salaires dans le secteur public sont fixes au cours du cycle (scenario 1) ou pro-cycliques et indexés sur les salaires 
du secteur privé (scenario 2). Utilisant une version de leur modèle structurel calibrée à l'économie américaine, ils prédisent que la volatilité de l'emploi est accrue par la présence d'un secteur public dans les deux scénarii, avec une plus grande amplification de la volatilité de l'emploi dans le premier scénario.

Horner et al. (2007) modélisent une économie dont le planificateur social bienveillant cherche à maximiser le bien être individuel avec, entre autres, les outils de politique d'emploi et de salaires publics. Cette économie est simulée avec et sans secteur public. Les auteurs montrent que la présence du secteur public a un effet ambigu sur le niveau global de l'emploi et que, dans des périodes d'incertitude accrue sur les conditions macroéconomiques, le niveau du chômage est plus élevé quand le secteur public existe. Cette prédiction s'explique par le fait que les agents averses au risque ont alors une préférence plus marquée pour les emplois plus sûrs du secteur public et y recherchent un emploi en plus grand nombre que dans des périodes plus stables du point de vue macroéconomique. Enfin, (2015) présente un modèle macroéconomique dynamique stochastique d'équilibre général avec des frictions d'appariement. En calibrant ce modèle sur des données américaines, il montre que de hauts salaires dans le secteur public pousse de trop nombreux chômeurs à chercher un emploi dans ce secteur, ce qui accroît le niveau du chômage. Il montre également que la cyclicalité des salaires publics a un fort impact sur la volatilité du taux de chômage.

A notre connaissance, notre travail (Bradley et al., 2015) est une des rares contributions proposant une modélisation explicite du comportement des entreprises du secteur privé en réponse à une politique des salaires publics, au sein d'un modèle d'appariement. Seuls les travaux d'Albrecht et al. (2015) et Burdett (2011) proposent des approches voisines. Albrecht et al. (2015) partent du modele canonique de Diamond-Mortensen-Pissarides (voir, par exemple, Pissarides, 2000) et y incluent un secteur public qui affiche un nombre exogène de postes vacants. Leur modele génère une dispersion des salaires dans le secteur privé grâce aux hypothèses de productivité spécifique à tout appariement employeurtravailleur et d'hétérogénéité du capital humain parmi les travailleurs. Le travail de Burdett (2011) est plus proche du notre au sens où les entreprises affichent des salaires plutôt que de négocier sur le partage du surplus et le modèle inclut un comportement de recherche en emploi. Ce dernier point, et la possibilité pour les travailleurs de changer d'emploi entre secteurs, est crucial pour capturer l'étendue de la concurrence entre le secteur public et le secteur privé sur le marché de l'offre de travail. Notre approche se distingue de celle de Burdett en ceci que nous modélisons la politique de salaires public par une distribution exogène de salaires offerts par le secteur public alors que Burdett fait l'hypothèse d'un salaire unique dans ce secteur. Ceci nous permet de prédire une réponse optimale du secteur privé qui consiste à afficher une distribution continue de salaires dans les postes vacants, ce qui nous conduit à illustrer les différences de salaires entre les deux secteurs. Enfin, notre modélisation laisse la possibilité d'une différence entre les emplois dans les deux secteurs en matière de sécurité de l'emploi. L'ensemble de ces hypothèses vise à 
reproduire de la manière la plus riche possible les multiples facettes des différences entres secteurs, tout en préservant notre souci de parcimonie.

Un aspect important de notre contribution (Bradley et al., 2015) est que nous estimons les différents paramètres de notre modèle et sommes ainsi en mesure de proposer des simulations de politiques contrefactuelles en termes d'emploi et de salaires publics. Une méthode similaire a été dévelopée par Meghir et al. (2015) pour analyser les interactions entre les secteurs formel et informel au Brésil. Dans leur modèle, les entreprises choisissent de manière endogène d'opérer dans le secteur formel ou informel en considérant les différences de règlementation et de prélèvements fiscaux dans les deux secteurs, ainsi que la probabilité et le coût d'une pénalité en cas de condamnation pour activité illégale.

\section{Différences statiques}

\section{a. Nos données}

Les bases de données que nous avons utilisées sont le BHPS (British Household Panel Survey) et le ECHP (European Community Household Panel). Nos échantillons de travail ainsi que les différentes sélections faites pour obtenir ceux-ci sont décrits dans PostelVinay et Turon (2007) et Dickson et al. (2014). Nous travaillons sur un échantillon $\mathrm{d}$ 'hommes en âge de travailler et qui ont des emplois à temps plein. Ce choix nous permet de faire abstraction des décisions de participation au marché du travail, de choix des heures travaillées, et de départ à la retraite. Les pays pour lesquels nous disposons de tailles d'échantillon suffisantes pour notre analyse sont la Grande-Bretagne, la France, I'Allemagne, les Pays-Bas, I'Italie et I'Espagne. La Table 1 donne un aperçu des tailles d'échantillon par pays, du nombre d'observations par individu et de secteur d'activité initial.

\section{b. Différences brutes de niveaux et de dispersion des salaires}

Les différences brutes de salaires entre les secteurs public et privé dans les six pays de notre échantillon sont présentées dans la Table 2 . II s'agit ici de salaires instantanés, c'est-à-dire de salaires mesurés à un instant donné, sans tenir compte des possibles variations de ce salaire autour de cet instant. Les chiffres reportés dans ce tableau réfèrent aux logarithmes de revenus bruts mensuels du travail. Ces différences brutes ne tiennent pas compte non plus des différences de composition des groupes d'employés du privé et du public en termes d'éducation et d'expérience. Comme nous le verrons plus tard, ces expliquent une part importante des écarts que nous observons dans cette table.

On observe dans la Table 2 que la prime salariale dans le secteur public est positive en moyenne dans tous les pays de notre échantillon. En France, cette prime se monte à 14\% 
du salaire. Elle varie de quelques points de logarithme ${ }^{2}$ en Allemagne, les Pays-Bas et I'Italie ( 4,9 et 10 respectivement) à des niveaux plus élevés en Grande-Bretagne (13\%) et en Espagne (31\%). D’autre part, la variance de ces données salariales est plus grande dans le secteur privé que dans le secteur public dans tous les pays de notre étude.

\section{c. Caractéristiques}

Un examen rapide de la composition de l'emploi dans les deux secteurs montre que les différences de caractéristiques des employés du secteur public relatives à celles du secteur privé expliquent en partie la prime salariale brute offerte par le secteur public. En effet, les employés du secteur public ont, en moyenne dans tous les pays de notre échantillon, des niveaux d'éducation plus élevés que ceux du secteur privé. Le panel Européen ECHP inclut une mesure d'éducation standardisée suivant la classification ISCED 3 qui nous permet de comparer la distribution de capital humain entre secteurs et pays en termes de répartition en trois catégories: "élevé", qui correspond à tous les types d'éducation tertiaire, "moyen", qui correspond à la fin des études secondaires (postérieures à l'age limite d'éducation obligatoire), et "bas", qui correspond à une fin d'études antérieure à la fin du cycle secondaire. La Table 3 montre que la première catégorie (capital humain élevé) représente environ un quart de l'emploi global en France, Angleterre, Allemagne et Pays-Bas. Cette part s'élève à un tiers de l'emploi en Espagne, et ne représente que 10\% de l'emploi en Italie. On observe davantage de variation d'un pays à l'autre s'agissant de la troisième catégorie (capital humain bas). Celle-ci varie de 10\% en Allemagne à $43 \%$ en Espagne. Dans chaque pays, le secteur public comprend une proportion plus grande de travailleurs à capital humain élevé. Ceci est particulièrement vrai dans le cas de l'Espagne où cette proportion est doublée dans le secteur public relativement au secteur privé.

Par ailleurs, dans tous les pays, les employés du secteur public sont en moyenne plus âgés et dotés d'une expérience potentielle plus longue. Cet écart est le plus marqué dans les Pays-Bas et en Italie où les employés du secteur public ont en moyenne 2.6 et 2 années (respectivement) d'expérience potentielle en plus par rapport à leurs homologues du secteur privé. En France, les employés du secteur public ont 1.4 ans d'expérience de plus que leur homologues du privé.

Ce phénomène de sélection des employés plus éduqués et plus âgés dans le secteur public ${ }^{4}$ contribue à l'existence d'une prime "apparente" de salaires entre les deux secteurs. Lorsque

\footnotetext{
2 Comme nos données sont presentées en logarithmes, une différence de 0.01 , que nous dénommons 1 point de log, correspond à une différence proche de $1 \%$. Cette approximation est moins bonne pour des différences plus importantes, par conséquent nous reportons dans le texte la différence en pourcentage pour la France, la Grande-Bretagne et l'Espagne.

3 International Standard Classification of Education.

4 Ainsi que notre mesure de l'hétérogénéité non-observée, comme détaillé dans notre stratégie empirique, voir section $4 \mathrm{a}$.
} 
nous estimons notre modèle empirique qui tient compte de cette sélection, nos résultats montrent que la "vraie" prime du secteur public en matière de salaires instantanés est fortement réduite par rapport à la prime: en France, elle n'est plus que de $3 \%$ (contre $14 \%$ de prime apparente), ce qui signifie que la majorité de la différence de salaires entre secteurs reflètent en fait des effets de composition. Les emplois offerts par le secteur public nécessitent des qualifications et une expérience qui attirent "mécaniquement" de plus hauts revenus, sans pour autant que le secteur public paye plus que le secteur privé toutes choses égales par ailleurs puisque la prime "vraie" n'est que de $3 \%$. Dans les autres pays, cette prime vraie est de $3 \%$ en Grande-Bretagne, de $11 \%$ en Espagne, et tombe même à $-4 \%$ aux Pays-Bas (voir Table 4 , colonne des différences en termes de prime "vraie"). Par "vraie" prime, nous entendons une différence qui affecterait chaque individu de notre échantillon si l'on simulait son salaire et sa trajectoire de salaire dans chacun des deux secteurs. Par contraste, la prime "apparente" reflète une différence moyenne de salaires ou de trajectoire de salaires entre le groupe d'individus de l'échantillon effectivement observés dans le secteur public et le groupe d'individus effectivement observés dans le secteur privé.

\section{Stratégie empirique et différences dynamiques}

Comme mentionné plus haut, nous considérons que ces différences statiques entre la valeur de l'emploi dans chaque secteur n'est qu'une facette parmi d'autres de l'attrait relatif d'un emploi dans le secteur public ou privé du point de vue des travailleurs. Les autres dimensions qui ont vraisemblablement de l'importance pour des agents à anticipations rationnelles sont la volatilité et la croissance des salaires ainsi que les taux de probabilité de perte d'emploi et de mobilité vers un autre emploi, au sein du même secteur ou avec changement de secteur.

\section{a. Stratégie empirique}

Notre stratégie empirique est décrite en détail dans Postel-Vinay et Turon (2007) et Dickson et al. (2014). Elle tire partie de la structure en panel de nos données pour incorporer toutes les dimensions de différences entre les emplois publics et privés mentionnées ci-dessus. Notre but est d'estimer simultanément les transitions entre chômage, emploi public et emploi privé et les trajectoires de revenus en emploi au sein d'un même secteur ou avec changement de secteur. La vraisemblance d'une série d'observations individuelles est conditionnelle, non seulement à une hétérogénéité observée capturant l'effet de d'éducation et de l'expérience, mais aussi à une hétérogénéité non-observée. Cette dernière est modélisée sous la forme de deux effets individuels fixes, l'un capturant une propension à l'emploi dans le secteur public et l'autre une propension à occuper un emploi à revenu plus élevé et/ou plus volatile. La dynamique du statut dans l'emploi et du secteur d'emploi est représentée par un processus markovien de premier ordre, tandis que la dynamique des salaires est représentée par un processus 
de Markov de deuxième ordre. Notre utilisation des deux termes d'hétérogénéité nonobservée constants nous permet de reproduire la persistance de l'emploi public, du chômage, et des revenus observés dans les données sans avoir à recourir à des processus markoviens à mémoire plus longue. Notre choix entre l'ordre des processus markovien et le nombre de classes d'hétérogénéité a été dicté par la taille de notre échantillon en coupe et en série temporelle ainsi que par notre objectif de reproduire les données de manière suffisamment fiable, particulièrement en matière de dynamique d'emploi et de salaires, pour être en mesure d'obtenir de bonnes prédictions en termes de valeurs actualisées (voir la discussion de la section 5 ).

\section{b. Sécurité de l'emploi}

Un résultat surprenant est que, lorsqu'on tient compte des effets de sélection dans le secteur public, la prime qu'offre celui-ci en matière de sécurité de l'emploi disparaît ou tout du moins s'amenuise considérablement. Ceci s'oppose à l'idée omniprésente dans le débat public selon laquelle les employés du secteur public bénéficient d'une sécurité de l'emploi bien plus grande que celle que ces mêmes employés pourraient trouver dans le secteur privé. En effet, les différences moyennes de taux de pertes d'emplois observées dans les données brutes reflètent principalement des effets de sélection, en ceci que des individus dont la propension à perdre un emploi est plus faible ont tendance à être employés dans le secteur public. Notre estimation de la "vraie" différence de taux de perte d'emploi est obtenue en comparant des trajectoires d'emploi simulées dans chaque secteur pour chaque individu de l'échantillon, qu'il soit ou non observé dans ce secteur. Le taux simulé moyen de perte d'emploi dans le secteur public reflète donc la propension à perdre son emploi de l'ensemble des travailleurs de l'échantillon dans la colonne de la Table 4 intitulée "différences vraies" alors que le taux de perte d'emploi dans le secteur public reporté dans la colonne "différences apparentes" reflète le taux moyen de perte d'emploi parmi les travailleurs effectivement observés dans le secteur public dans notre échantillon.

En France, les taux de perte d'emploi parmi les travailleurs effectivement observés dans chaque secteur sont $0.04 \%$ et $0.02 \%$ mensuels respectivement dans les secteurs privé et public. En revanche, une fois que les effets de sélection sont pris en compte et que nous simulons les trajectoires d'emploi pour chaque individu, nous estimons un taux moyen de perte d'emploi dans le secteur privé de $0.05 \%$ et de $0.04 \%$ dans le secteur public. Le "vrai" avantage en termes de sécurité de l'emploi en France est donc beaucoup moins important que l'avantage "apparent" qui ne prend pas en compte le fait que les employés que nous observons dans le secteur public ont une tendance intrinsèque à conserver leur emploi sur une plus longue durée que les employés que nous observons dans le secteur privé. En Allemagne, la prime positive de sécurité de l'emploi dans le secteur public qui apparaît dans les données brutes est inversée lorsque nous estimons des effets individuels: si tous les individus de notre échantillon comparent un statut d'employé dans le secteur et le secteur public (dont au moins un est contrefactuel dans les données), l'écart moyen de taux de risque de perte d'emploi est en fait plus élevé de $3 \%$ dans le secteur public. 


\section{c. Dynamique des salaires}

Les rendements de l'expérience sont plus grands dans le secteur public en France et en Allemagne où la différence s'élève à 2 et 5.4 points de logs par an, respectivement. En revanche, en Italie, les rendements de l'expérience sont plus bas de 6.3 points log annuels dans le secteur public. En Espagne, la différence est négative (en faveur du secteur privé) mais n'est pas significative. En Grande-Bretagne, le rendement de l'expérience est similaire dans les deux secteurs.

S'agissant de la volatilité, mesurée par la première auto-covariance des salaires et une fois les effets de sélection pris en compte au moyen de notre stratégie empirique, nous estimons que les salaires sont plus persistants dans le secteur public pour l'Italie, l'Espagne et la Grande-Bretagne. D'autre part, la variance des salaires est plus faible dans le secteur public dans tous les pays sauf la France et l'Italie, indiquant une plus grande variance des chocs transitoires sur les salaires dans le secteur privé. Dans ces deux dernières dimensions, "persistance" et "dispersion des salaires", les effets de sélection sont modestes, alors qu'ils sont relativement conséquents dans la dimension "rendement de l'expérience". 


\section{Valeurs actualisées}

\section{a. Agrégation}

Comme nous l'avons vu dans la section précédente, les différences intersectorielles ne sont qu'imparfaitement mésurées par les différences de salaires en coupe puisque des différences de dynamiques de salaires et de sécurité de l'emploi existent également. Pour des agents à anticipations rationnelles, ces différences dynamiques ont de l'importance et doivent être prises en compte si l'on essaie d'évaluer l'attrait relatif de l'emploi dans les deux secteurs pour ces agents. II est par conséquent nécessaire d'agréger les différences public-privé dans ces quatre dimensions: salaires en coupe, rendement de l'expérience, persistance et dispersion des salaires, et sécurité de l'emploi pour quantifier cet attrait relatif de l'emploi public. C'est la comparaison que nous faisons ici au moyen de "valeurs actualisées" définies dans la section suivante.

\section{b. Construction}

La mesure de valeur actualisée que nous utilisons est simplement une somme actualisée d'une trajectoire de revenus dans un secteur donné pour un individu donné au cours de sa carrière et de sa retraite. Ces trajectoires de revenus sont obtenues grâce à des simulations, pour tous les individus de notre échantillon, de statut dans l'emploi (chômage, emploi dans le secteur public, emploi dans le secteur privé) et de revenus. Ces simulations sont effectuées avec les coefficients obtenus lors de notre estimation de notre modèle dynamique décrit dans les sections précédentes. Notre confiance quant à l'utilisation de ces coefficients poursimuler des trajectoires longues s'appuie sur le très bon ajustement statistique de notre modèle aux données, aussi bien dans les aspects statiques des distributions de variables en coupe que dans les dimensions dynamiques de trajectoires observées de statut dans l'emploi et de revenus.

Afin de simuler les revenus dans la retraite, nous faisons l'hypothèse que celle-ci consiste en un revenu constant, égal a une fraction calibrée à $40 \% 5$ du dernier revenu dans l'emploi, et perçu pendant une durée de vie restante calibrée à 20 ans. Nous fixons le facteur annuel d'escompte à $95 \%$ et le niveau d'expérience accumulée à l'âge de la retraite à 45 ans. Bien entendu, ces valeurs sont plus en adéquation avec les données observées sur les conditions de la retraite dans certains pays que dans d'autres. Nous choisissons néanmoins de fixer ces paramètres à des valeurs communes pour les six pays de notre échantillon afin de de préserver un cadre commun pour notre évaluation de la prime du secteur en termes de valeurs actualisées. On pourrait également interroger notre hypothèse d'uniformité de l'âge de départ à la retraite et du ratio de remplacement de la retraite dans les secteurs public et privé, ce qui s'oppose aux données. Afin d'évaluer l'importance de ces hypothèses,

\footnotetext{
${ }^{5}$ Ceci peut paraître très bas relativement au système de retraites en France. Notre calibration à $40 \%$ est un compromis entre les valeurs de ce ratio de remplacement dans les différents pays de notre échantillon.
} 
nous avons procédé à l'estimation des primes de long-terme (comme détaillé ci-dessous) sous l'hypothèse alternative de paramètres de retraite spécifiques à chaque pays et secteur d'emploi. L'impact de ces changements s'avère négligeable dans presque tous les cas. Ceci conforte notre choix de paramètres de retraite communs, qui nous permet de montrer plus clairement l'effet des différences de dynamiques de salaires et d'emploi sur les primes de long terme dans les différents pays. D'autre part, les paramètres de retraite ont peu d'impact sur les primes de long-termes car les différences de revenus dans la retraite qu'ils créent sont négligeables du point de vue d'individus en début et en milieu de carrière raisonnant sur des valeurs actualisées.

Une autre hypothèse sous-jacente à nos simulations de trajectoires de long terme consiste à supposer que l'environnement économique est stable en ce qui concerne toutes les dimensions de notre modèle, c'est-à-dire les distributions de salaires en coupe, les dynamiques des salaires, les taux de transition entre secteurs d'emploi et le poids du secteur public dans l'économie. Bien sûr, cette hypothèse peut paraître forte, particulièrement à un horizon très long. Cependant, pour l'individu qui évalue une espérance de prime de long terme dans le secteur public aujourd'hui, l'hypothèse de stabilité s'impose comme un choix naturel en l'absence d'information sur la nature d'un éventuel changement aux paramètres pertinents.

Nous effectuons nos simulations de trajectoires de revenus en l'absence de mouvement entre secteurs d'emploi, chômage compris. Ceci nous permet d'évaluer l'impact des différences de salaires en coupe et de dynamique entre les secteurs public et privé sur la prime du public sur le long terme, ainsi que l'impact de la sélection des individus entre les secteurs sur cette prime. Plus précisément, pour chaque individu de notre échantillon, nous simulons une trajectoire de revenus dans les cas extrêmes où sa carrière future se déroulerait exclusivement dans l'un ou l'autre secteur. La différence des valeurs actualisées ainsi obtenue est ce que nous appellerons la "vraie" prime de long-terme du secteur public. Nous employons ce terme pour indiquer qu'une telle prime fait abstraction des effets de sélection et représente la moyenne sur notre échantillon des différences (potentielles) de valeurs actualisées de trajectoires de revenus pour chaque individu dans chaque secteur. Nous contrasterons, dans les résultats présentés ci-dessous, cette "vraie" prime de longterme à une prime "apparente" de long-terme qui ne tient pas en compte les effets de sélection. Cette dernière mesure la différence moyenne entre, d'une part, les valeurs actualisées de trajectoires de revenus dans le secteur public pour les individus observés dans le secteur public à la date de l'échantillon et, d'autre part, les valeurs actualisées de trajectoires de revenus du secteur privé pour les individus effectivement observés dans les secteur privé. La prime 'apparente' ne reflète donc pas une différence entre deux trajectoires contrefactuelles pour le même individu puisqu'elle amalgame une telle différence avec la différence de composition des groupes d'individus employés dans les deux secteurs. 


\section{c. Prime du secteur public en termes de valeurs actualisées}

Afin d'illustrer l'importance des différences de dynamiques des revenus au sein de la prime de long terme, nous allons comparer nos résultats sur les primes en valeurs actualisées aux primes en termes de salaires instantanés (en coupe). Le contraste qui en résulte justifie notre argument selon lequel les différences de salaires en coupe ne donnent qu'une image tronquée de la différence des valeurs des emplois dans les secteurs public et privé pour des agents à anticipations rationnelles.

Dans les Tables 4 et 5 , nous présentons les primes en question à plusieurs endroits de chaque distribution puisque ces distributions ne sont pas identiques. Une première observation est que, conformément à nos attentes, les primes de valeurs actualisées sont très différentes des primes de salaires instantanés. Deux raisons pour lesquelles les primes de long terme diffèrent de la moyenne des primes instantanées sur le cycle de vie alors que nous sommes dans un environnement supposé stationnaire sont que les valeurs de long terme incluent la valeur de la retraite alors que les données en coupe ne concernent que les individus en âge de travailler, ce qui magnifie les effets de différences de rendements de l'expérience, et que, dans les données en coupe, les revenus des travailleurs au chomage ne sont pas affectés à la distribution des revenus d'aucun secteur alors que c'est effectivement le cas lors d'un calcul de valeur actualisée où l'individu anticipe d'être sans emploi à certains moments de sa carrière future. Si l'on suppose que les primes de long terme sont plus pertinentes pour des agents à anticipations rationnelles, notre contribution nuance alors fortement la manière dont les différences de conditions salariales dans l'emploi public et prive sont habituellement perçues. Pour résumer, les différences de dynamiques de salaires que nous estimons ont tendance à atténuer, dans la majorité des cas --quoique pas en France--, les différences statiques de salaires qui sont normalement au coeur du débat sur les différences public-privé. Notre argument consiste à dire que l'attention portée sur ces différences statiques ne se justifie que dans un contexte où les agents seraient myopes. En présence d'anticipations rationnelles, les individus comparent les valeurs actualisées, et le débat public devrait par conséquent se concentrer aussi sur ces différences de long-terme.

Nous procédons ensuite à un examen plus détaillé de ces différences de long-terme (voir Tables 5 et 6). Dans la mesure où les distributions de salaires et de valeurs actualisées ne sont pas identiques dans les deux secteurs, il est utile de décrire les primes de long-terme en plusieurs points de la distribution plutôt que simplement à la moyenne. En France, comme les différences de mobilité des salaires et de sécurité de l'emploi sont assez faibles entre les secteurs, la prime "vraie" en valeurs actualisées est en fait assez proche de la prime "vraie" en salaires instantanés, à $+6 \%$ contre $+3 \%$. Ceci signifie qu'un emploi du secteur public Français offre une valeur de long terme qui est $6 \%$ supérieure à la valeur d'un emploi dans le secteur privé que le même individu pourrait occuper. Cette prime fait donc 
abstraction des effets de composition et correspond bien à un effet potentiel (moyen) pour chaque individu de notre échantillon.

En Italie, la "vraie" prime de long-terme dans le secteur public, c'est-à-dire en prenant en compte les effets de sélection, est estimée à - 2 points de log au 1oeme pourcentile et n'est pas significative, alors que la différence de salaires statiques au même point de la distribution est positive et significative à 8 points de log. En Espagne, les résultats sont similaires : la "vraie" prime publique de long terme est de 8 points de log au 1oeme pourcentile alors qu'elle est de 18 points de logs lorsque l'on regarde la "vraie" prime de salaires instantanés. Une explication de ces différences entre primes de long-terme et prime instantanée dans le bas de la distribution tient aux différences de dynamique de salaires entre les deux secteurs. Dans le secteur public, les salaires ont tendance à être plus persistants que dans le secteur privé. Pour les individus à salaires faibles, ceci pénalise l'espérance des salaires futurs et ainsi la valeur actualisée de l'emploi dans le secteur public relative à celle du secteur privé où le salaire bas est moins persistant en moyenne. De plus, en Italie et en Espagne, les rendements de l'expérience estimés sont plus faibles dans le secteur public. En revanche, en haut de la distribution des valeurs actualisées, ces deux écarts de dynamiques (persistance des salaires et rendements de l'expérience) se compensent, si bien que les primes du secteur public de long-terme et de salaires instantanés sont très similaires au goème pourcentile.

En revanche, en Allemagne, les différences dynamiques viennent accentuer de 5 points de log les différences estimées dans le cadre statique car les rendements de l'expérience du secteur public y sont estimés supérieurs à ceux du secteur privé, alors que les différences de persistance des revenus y sont négligeables.

En Grande-Bretagne, la "vraie" prime de long-terme dans le public secteur varie entre 3 à 10 points de log dans les deux premiers (plus bas) déciles de la distribution et devient nulle dans le haut de la distribution, tandis que la "vraie" prime en termes de salaires instantanés va de 20 points de log dans le bas de la distribution à une prime négative de 4 a 10 points de log dans les deux déciles les plus hauts. Dans Postel-Vinay et Turon (2007) nous montrons que ce contraste entre les deux primes s'explique par le fait que la variance des chocs transitoires est plus grande dans le secteur privé que dans le secteur public. Ceci accroît relativement la variance des salaires instantanés dans le privé même si, sur le long terme, cette différence de variances s'estompe largement du fait de la nature transitoire de ces chocs. Les rendements de l'expérience sont les mêmes dans les deux secteurs dans ce pays.

Parmi nos résultats sur la "vraie" prime de long-terme, nous observons dans la Table 6 que cette prime est conséquente dans certains pays et en certains points de la distribution. En France et en Espagne, les primes de long-terme sont positives dans toute la distribution, particulièrement dans la moitie basse, allant de 6 (respectivement 7) points de log au 
niveau de la médiane à 9 (respectivement 8) points de log dans le bas de la distribution. En revanche, nous observons une prime de long-terme négative dans les Pays-Bas dans le haut de la distribution, où la valeur actualisée de l'emploi dans le secteur public est inferieure à celle de l'emploi dans le secteur privé de 5 points de log.

Pour ce qui est des inégalités au sein de chaque secteur, nous comprenons, au vu des résultats ci-dessus, que la dispersion des salaires instantanées n'est pas une bonne mesure des inégalités de long-terme puisque nous observons non seulement des composantes dynamiques des processus de salaires, mais aussi des différences substantielles entre ces comportements dynamiques entre différents points de la distribution et entre secteurs. Encore une fois, pour des agents à anticipations rationnelles, une mesure de la dispersion des valeurs a long-terme reflète mieux l'étendue de l'inégalité de revenus que la dispersion des salaires instantanés. Comme mentionné ci-dessus, nos résultats pour la GrandeBretagne montrent que la plus grande dispersion des salaires instantanés dans le secteur privé est due en très grande partie au fait que la variance des chocs transitoires y est plus élevée. Nous estimons un phénomène similaire en Espagne. En France et en Italie en revanche, les parts de la variance totale des salaires expliquées par les composantes transitoires et permanentes des salaires sont similaires dans les deux secteurs. Enfin, en Allemagne et aux Pays-Bas, les salaires instantanés et les valeurs de long terme ont une dispersion plus grande dans le secteur privé, ce qui suggère une plus grande égalité des conditions salariales sur le court et sur le long termes dans le secteur public dans ces pays.

\section{Sélection}

Examinons maintenant la moitié droite de la Table 6 qui montre les primes du secteur public de long-terme et instantanée, mais sans tenir compte de la sélection non-aléatoire des individus entre secteurs. Tout d'abord, le contraste entre les "vraies" primes de la moitié gauche de la table et les primes "apparentes" de la moitié droite montrent que la sélection est positive dans tous les pays et à tous les niveaux de la distribution des valeurs actualisées. Par "sélection positive", nous entendons que les individus qui travaillent dans le secteur public présentent des caractéristiques moyennes corrélées avec des salaires et des valeurs de long-termes supérieures aux individus qui travaillent dans le secteur privé. Ceci était déjà apparent dans notre analyse descriptive des niveaux moyens d'éducation dans les deux secteurs (voir Table 3 et Section 3c). Nos résultats (voir Dickson et al., 2014, pour un compte rendu détaillé de ces résultats) montrent que la sélection des individus entre secteurs est positive aussi en termes d'hétérogénéité non-observée. Cette sélection positive est particulièrement marquée dans le haut de la distribution en France et en Italie.

Cette sélection non-aléatoire des travailleurs entre les deux secteurs, dans laquelle des individus dont les caractéristiques observées et non-observées sont corrélées avec des 
revenus plus hauts et des emplois plus stables, confirme des résultats déjà présents dans la littérature. Hartog et Oosterbeek (1993) et van Ophem (1993) pour les Pays-Bas, Dustmann et van Soest (1998) pour l'Allemagne, Disney et Gosling (1998) pour la GrandeBretagne et Bargain et Melly (2008) pour la France trouvent aussi des effets de sélection importants.

Nos résultats confirment ceux de Dustmann et van Soest (1998) qui estiment une prime négative pour les salaires instantanés du secteur public en Allemagne et qui s'amenuise avec l'âge. Nos résultats pour la France sont cohérents avec la conclusion de Bargain et Melly (2008) selon laquelle les primes salariales du secteur public sont plus importantes dans le bas de l'échelle des salaires. En France, les effets de sélection representes environ trois quarts de la prime apparente du secteur public.

\section{Un modèle structurel des interactions entre secteur public et secteur privé}

Au vu des résultats produits par nos travaux descriptifs (Postel-Vinay et Turon, 2007, Dickson et al., 2014) une question se pose naturellement : quels sont les mécanismes qui régissent les interactions entre les deux secteurs d'emploi? De quelle manière les deux secteurs rivalisent-ils pour l'offre de travail des individus? Pour répondre à ces questions, nous avons développé un modèle structurel du marché du travail incorporant un employeur public important et régi par des objectifs différents de ceux des entreprises privées (Bradley et al. 2015). Le modèle structurel a l'inconvénient de devoir simplifier certaines dimensions représentées de manière plus riche dans les travaux descriptifs, par exemple l'hétérogénéité des travailleurs. En revanche, si le modèle offre un bon ajustement statistique, il permet alors de simuler l'effet potentiel de politiques d'emploi et de salaires publics sur le marché du travail (emploi, distributions des salaires) grâce à la modélisation des mécanismes de réaction à ces politiques par les entreprises privées et les travailleurs. Il va sans dire que, conformément à l'argument exposé jusqu'ici, nous modélisons un environnement dynamique qui offre un rôle aux multiples dimensions des différences dans l'emploi dans chaque secteur: salaire moyen, rendement de l'expérience, volatilité des salaires et sécurité de l'emploi. La comparaison par les travailleurs d'offres d'emploi dans les deux secteurs repose alors sur une comparaison des valeurs actualisées des deux offres.

Notre modèle s'inscrit dans la littérature des modèles de recherche et d'appariement. II comprend un secteur public dont les outils de politique économique sont la distribution d'offres de salaires dans ce secteur et le niveau de l'emploi public, en proportion de l'offre de travail totale. A cette fin, le secteur public produit un effort de recrutement tel que les taux d'offres venant du secteur public pour les travailleurs est fixe -- et exogène, détermine 
par ce choix politique en terme de taille de l'emploi public -- et de protection de l'emploi (public), sous la forme d'un taux fixe de licenciement dans le secteur public. Le choix optimal de cette taille et des niveaux de salaires publics n'est pas modélisé dans notre travail. En effet, l'objectif du gouvernement s'agissant de la taille du secteur public se fonde sur des considérations qui dépassent les mesures du marché du travail auxquelles nous nous intéressons ici. Nous avons donc opté pour un travail confiné à l'analyse d'un choix politique exogène sur un marché du travail où l'allocation des travailleurs et les décisions des entreprises privées répondent de manière endogène à ce choix du gouvernement.

Les travailleurs sont supposés homogènes et neutres au risque. ${ }^{6}$ Ils peuvent être soit au chômage, soit en emploi, public ou privé. Tous sont à la recherche d'un (meilleur) emploi dans un marché du travail frictionnel. Cette recherche est considerée comme aléatoire, donc non dirigée vers un secteur particulier. Quelque soit leur état, ils reçoivent des offres selon un processus de Poisson, venant des deux secteurs. Comme les différents statuts dans l'emploi peuvent être associés à des intensités de recherche et/ou des efficacités de recherche différentes, nous faisons l'hypothèse que les taux d'arrivée d'offres venant d'un secteur donné ne sont égaux qu'à un facteur multiplicatif près (fixe) selon le statut du travailleur (chômeur, employé du secteur privé ou employé du secteur public). Les taux d'offres venant du secteur public dépendent du choix politique décrit ci-dessus. Les taux d'offres du secteur privé dépendent du choix optimal des entreprises privées, détaillée cidessous. Les travailleurs sont des agents à anticipations rationnelles et font leur choix d'accepter ou non une offre de travail venant d'un secteur précis, qui prend la forme d'un salaire fixe sur toute la durée du poste, sur la base de la valeur actualisée de l'emploi dans ce secteur et à ce salaire. Cette valeur comprendra donc l'anticipation par le travailleur de recevoir des offres extérieures aux taux d'arrivée correspondant à ce statut qui lui permettront de faire des gains en valeur dans le futur, ainsi que l'anticipation d'un licenciement à un horizon espéré déterminé par le taux de processus de Poisson des chocs de destruction de l'emploi, spécifique à chaque secteur. Nous faisons l'hypothèse que les caractéristiques d'un emploi hormis le salaire et sa progression espérée n'apportent pas de valeur au travailleur, à l'exception du secteur d'emploi. En pratique, nous modélisons le flux d'utilité d'un emploi du secteur public comme la somme du salaire instantané et d'une "aménité du secteur public". Notre application empirique révèle que cette aménité n'est pas statistiquement significative; nous poursuivons donc la discussion dans cette section

\footnotetext{
${ }^{6}$ Ces hypothèses sont simplificatrices et visent à préserver la parcimonie du modèle structurel. Bien-sûr, si le secteur privé offre des emplois plus volatiles en termes de sécurité et de salaires, celui-ci devra offrir des primes de salaires instantanés plus grandes que dans le modèle présenté ici pour débaucher un employé du secteur public. Aussi, pour reproduire les effets de sélection présentés dans les sections précédentes, il faudrait aggréger des sous-marchés de travailleurs homogènes de qualités différentes et faisant face à des conditions de marché (distributions de salaires, taux d'arrivée d'offre, taux de perte d'emploi) différentes. Ce travail, bien qu'intéressant, dépasse le cadre du projet présenté ici.
} 
sans plus mentionner cette aménité. Dans le modèle théorique, celle-ci vise à rendre compte de l'existence possible d'une stigmatisation ou d'une satisfaction intrinsèque à l'emploi dans le secteur public.

La comparaison par le travailleur entre les valeurs dynamiques du chômage et de l'emploi dans chaque secteur et à chaque niveau de salaire se traduit par des salaires de réserve pour toutes les transitions possibles. Plus précisément, le travailleur au chômage a un salaire de réserve pour chaque secteur au dessus duquel il va accepter une offre d'emploi dans ce secteur, le travailleur du secteur public/privé a des salaires de réserve au dessus desquels il acceptera une offre du secteur public/privé. Ces salaires de réserve dépendent du salaire que l'employé reçoit dans son poste actuel puisqu'il compare la valeur de l'emploi qu'il détient et la valeur de l'emploi alternatif qui lui est proposé. Si les deux emplois sont dans des secteurs différents, c'est-à-dire si le travailleur est actuellement employé dans le secteur public et reçoit une offre du secteur privé, ou inversement, les composantes dynamiques du poste actuel et du poste proposé diffèrent, si bien que le salaire de réserve pour accepter cette proposition sera différent du salaire actuel. Si les composantes dynamiques du poste proposé sont plus avantageuses, par exemple si les taux d'offres extérieures y sont plus élevés, ou si le taux de licenciement y est plus faible, le salaire de réserve sera inférieur au salaire actuel. Bien entendu, quand l'offre extérieure et le poste actuel sont dans le même secteur, ces composantes dynamiques sont identiques, donc le salaire de réserve est égal au salaire actuel.

Pour des taux d'arrivée d'offres et de licenciement et des distributions de salaires offerts donnés, les règles de décisions simples exposées ci-dessus déterminent les mouvement des travailleurs entre les différents statuts dans l'emploi, les comportements dynamiques de leurs salaires, et les distributions de salaires en coupe dans chaque secteur à l'équilibre.

Examinons a présent le comportement des entreprises privées. Nous faisons l'hypothèse qu'il existe une mesure unitaire d'entreprises privées dont l'objectif est de maximiser le profit. Ces entreprises sont hétérogènes en termes de productivité, et vont choisir un niveau de salaire et un taux de recrutement optimaux en fonction de cette productivité. Comme nous l'avons vu dans les paragraphes précédents, le niveau de salaire dans l'entreprise, qui doit être le même pour tous les employés de cette entreprise, détermine le taux auquel les employés quittent l'entreprise pour accepter des offres extérieures (nous sommes dans un environnement de salaires affichés ou les contre-offres ne sont pas possibles). D'autre part, recruter à un taux donné entraîne un coût pour l'entreprise (affichage de vacances, sélection, entretiens et intégration des candidats) qui dépend du taux d'embauche de manière convexe. L'impact de ces mécanismes sur le profit de l'entreprise détermine les niveaux optimaux de salaire et de recrutement en fonction de sa productivité. Quand ce comportement individuel est agrégé sur la population des entreprises privées, nous obtenons un taux d'offre total venant du secteur prive (rappelons 
que la recherche d'emploi est aléatoire) et une distribution des salaires offerts par ce secteur.

Cette modélisation du comportement des entreprises privées "boucle" le modèle et nous permet d'endogénéiser la réponse du secteur privé à la politique publique d'emploi et de salaires. Dans Bradley et al. (2015), nous estimons ce modèle structurel sur des données de panel britanniques sur la période 2004-2008 stratifiées en quatre groupes de genre et de niveau de qualification. Nous obtenons un bon ajustement statistique qui nous permet d'envisager des simulations de politiques publiques contrefactuelles avec confiance. Les paramètres que nous estimons conduisent aux observations suivantes: une dominance stochastique de la distribution des offres du salaire privé par la distribution des offres du secteur public, une plus grande dispersion des salaires offerts dans le secteur privé, et une plus grande mobilité (ascendante) des salaires dans le secteur privé. Une conséquence de cette dernière observation est que le salaire de réserve des employés du secteur privé pour accepter une offre du secteur public est supérieure au salaire actuel, particulièrement dans le bas de la distribution des salaires. En effet, la plus grande sécurité de l'emploi public, une différence que nous estimons être modeste dans notre échantillon (qui relate à la Grande Bretagne, mais nous avons vu plus haut que les différences "vraies" en terme de sécurité de l'emploi sont faibles dans tous les pays de notre échantillon Européen), ne compense pas l'attrait d'une plus grande facilité à gravir l'échelle des salaires dans le secteur privé.

\section{Simulations de politiques contrefactuelles}

Nous sommes maintenant en mesure de simuler l'impact potentiel d'un changement de politique d'emploi et/ou de salaires dans le secteur public. Pour cet exercice, il est essentiel de disposer d'un modèle structurel dont la capacité à reproduire les moments estimés à partir des données a été établie. Ceci nous permet de prédire, avec un degré de fiabilité satisfaisant, des mécanismes crédibles de comportements des différents agents dans l'économie (travailleurs et entreprises privées), et ainsi leurs réactions à de nouveaux paramètres régissant les salaires, taux d'emploi ou taux de licenciement dans le secteur public. Afin de contribuer au débat politique actuel sur les coupes budgétaires, nous consacrons notre travail de simulations à l'évaluation de différentes politiques de réduction de la masse salariale du secteur public. Clairement, plusieurs choix s'offrent au décideur politique pour atteindre cet objectif : réduire les salaires des employés de ce secteur, uniformément ou de manière progressive, réduire le taux d'embauche, ou procéder à des destructions d'emplois dans ce secteur. Il est utile de préciser que nous n'avons pas l'ambition de comparer la politique de coupes budgétaires par rapport à des alternatives de choix macroéconomiques. Nous nous cantonnons à comparer les impacts des choix 
possibles pour effectuer ces coupes budgétaires dans la masse salariale publique, conditionnellement à avoir fait ce choix politique.

Au Royaume-Uni, la masse salariale du secteur public se montait à 174 milliards de livres, soit environ $30 \%$ de la dépense publique (ONS, 2009). Dans un contexte de restrictions budgétaires, la question posée est donc celle d'une réduction de cette masse salariale de la manière la moins défavorable au marché du travail.. Les différentes options politiques que nous simulons illustrent de manière stylisée les choix faits en Italie, Royaume-Uni, Espagne et Portugal. En effet, ces pays ont adopté des tactiques très différentes dans une optique partagée de réduire la dépense publique en termes de salaires. En Italie, les recrutements du secteur public ont été suspendus ; au Royaume-Uni, une vague de mises à pied dans le secteur public a eu lieu ; en Espagne, tous les salaires du secteur public ont été bloqués ; au Portugal, les employés du secteur public les mieux rémunérés ont subi des baisses de salaires.

Quelque soit le choix politique adopté, une conséquence immédiate des changements dans le secteur public pour réduire la masse salariale est de rendre le secteur public moins attractif que le secteur privé. Ce dernier bénéficie donc d'une réduction de la concurrence pour l'offre de travail : le taux de contact avec un travailleur, chômeur ou employé, augmente, de même que le taux d'acceptation des offres, tandis que le taux de perte d'employés au profit du secteur public décroit. II devient donc plus profitable pour les entrepreneurs privés de recruter. Une conséquence de cet accroissement des embauches dans le secteur privé est qu'il accroît les mouvements de main d'oeuvre au sein du secteur privé : les employés démissionnent plus souvent de leur position actuelle pour accepter une meilleure offre dans le même secteur. Dans nos simulations, cet aspect des mesures d'austérité, même s'il n'est pas recherché par la puissance publique s'avère substantiel. Notons que cet effet est absent dans les modèles standards du marché du travail qui ignorent la réallocation directe des travailleurs d'emploi à emploi. D'autre part, la moindre compétition exercée par le secteur public a un effet négatif sur le choix optimal de salaires offerts par les entrepreneurs prives créant de nouveaux emplois. La distribution d'offres de salaires privés est donc en moyenne plus basse qu'avant la politique d'austérité. Toutefois, l'impact final de cette politique sur les salaires dans l'emploi privé est minime car la réduction des salaires offerts à l'embauche est compensée par un accroissement de la vitesse à laquelle les employés peuvent gravir l'échelle des salaires dans le secteur privé du fait d'un taux d'arrivée des offres plus élevé.

En résumé (le détail de ces simulations figure dans Bradley et al., 2015), nos simulations montrent que les quatre choix de politiques de réduction budgétaires ont des effets agrégés qualitativement similaires : un accroissement des embauches dans le secteur privé et du mouvement des travailleurs au sein de l'emploi privé par le biais de la recherche en emploi, une réduction de l'emploi public en grande partie compensé par la hausse de l'emploi dans le secteur privé, et très peu d'impact sur le niveau du chômage. Dans tous les 
cas, l'impact sur les salaires moyens du secteur privé est négligeable alors que ceux du secteur public sont réduits en moyenne de $1 \%$ à $8 \%$ selon les politiques choisies.

Trois des options ouvertes au secteur public en termes de politique d'emploi et de salaires -- accroissement des mises à pied, diminution des embauches, diminution uniforme des salaires -- ont des effets très similaires sur les quatre groupes de travailleurs et n'affectent pas la composition de l'emploi dans chaque secteur. En revanche, la politique de diminution des salaires progressive, simulée par une réduction des salaires des deux groupes (femmes et hommes) de travailleurs qualifiés seulement, nécessite une baisse des salaires bien plus forte pour les groupes concernés, qui ne représentent environ que $30 \%$ de la main d'œuvre, que dans les trois autres scenarii conçus pour atteindre le même objectif global de diminution de masse salariale donnée. Ceci accentue l'impact de ce choix politique sur les travailleurs plus qualifiés et conduit à une réduction substantielle de la part des employés qualifiés dans le secteur public (et à un accroissement de cette part dans le secteur privé). Comme nous ne modélisons pas la fonction de production du secteur public en fonction des quantités de main d'oeuvre qualifiée et non qualifiée, nous ne sommes pas en mesure d'évaluer le coût de ce changement de la composition de la main d'oeuvre dans le secteur public. II est cependant probable que ce coût existe puisque l'activité de ce secteur est concentrée dans un certain nombre de produits et services caractérisés par un coefficient élevé de compétence, et qui n'ont pas de substituts proches produits dans le secteur privé. Cet argument s'accorde avec le résultat obtenu par Gomes (2016) au moyen d'un modèle différent du nôtre et calibré à l'économie britannique : il estime qu'un choix politique de réduction des salaires qualifiés de $6 \%$ déteriore le bien-être global. 


\section{Institutions du secteur public}

Avant de conclure, il nous semble utile d'inscrire les résultats présentés dans ce travail dans le contexte institutionnel propre à chaque pays. En effet, des différences de pratiques de fixation des salaires, de types de contrats, d'examens d'entrée, de carrières proposées, et de conditions de retraite entre les secteurs peuvent toutes mener à des niveaux et à des dynamiques de salaires différentes entre public et privé, et par là même modifier les primes de long-terme dans le secteur public.

En terme de fixation des salaires, le secteur privé obéit à un objectif de maximisation des profits tandis que le secteur public répond à des considération d'ordre budgétaires et politiques. Le pouvoir des syndicats, la portée des accords collectifs de salaires, et la possibilité de mesurer la productivité des travailleurs individuels sont autant de facteurs qui peuvent causer des différences salariales entre les secteurs. En outre, le gouvernement peut décider, pour des raisons d'ordre politique, de verser à ses employés peu qualifiés des salaires élevés relativement au secteur privé, et se montrer réticent à payer les niveaux de salaires prévalant dans les plus hauts pourcentiles de la distribution du secteur privé. Un emploi dans le secteur public est dans certains cas, particulièrement pour les employés ayant un statut de fonctionnaire, associé à certains privilèges ainsi qu'à un statut particulier. Par conséquent, la rémunération, surtout pour les hauts fonctionnaires, ne consiste pas uniquement en un niveau de salaire.

Il est utile de noter que, malgré plusieurs vagues de privatisations et de réformes variées, le montant de la charge salariale dans le secteur public reste stable relativement au PNB de chaque pays dans la fenêtre temporelle de notre échantillon (1994-2001). Bien que des changements institutionnels destinés à augmenter la concurrence et l'efficacité dans le secteur public aient été introduits dans les années go dans plusieurs pays Européens, les règles qui déterminent les salaires dans les deux secteurs n'en demeure pas moins clairement différentes (voir Giordano et al., 2011, pour plus de détails). Du Caju et al. (2008) comparent les modes de fixation des salaires du secteur privé entre les pays et concluent que les Pays-Bas, la France, l'Allemagne et I'Italie ont tous un système règlementé de négociation des salaires, qui se caractérise par un haut taux de couverture des accords collectifs de salaires, de négociations salariales sectorielles, et d'une absence de coordination autre que par le niveau du salaire minimum -- une règlementation qui n'est présente qu'en France et aux Pays-Bas. L'Espagne a aussi un salaire minimum national, et le gouvernement y joue un rôle important dans la fixation des salaires. Le Royaume-Uni, en comparaison à ces pays, a un marché du travail relativement peu règlementé.

Dans tous les pays de notre échantillon, les salaires du secteur public sont fixés a l'échelle nationale -- au moins pendant notre période d'observation. L'Allemagne, l'Italie, l'Espagne et la France ont un système de fixation de salaires des fonctionnaires très rigide et 
déterministe en fonction des qualifications, du niveau hiérarchique et du poste occupé. Dans le secteur privé, la France a cette particularité que les négociations salariales au niveau de l'entreprise ont plus d'importance que les accords par industrie ou par secteur d'activité (voir Broughton, 2009 et Commission Européenne, 2013).

En France, en Allemagne et en Italie, une distinction nette existe entre les conditions d'emploi pour les fonctionnaires d'Etat et le reste des employés du secteur public. Cette distinction a trait aux échelles salariales, à la protection contre le licenciement et au régime de retraite. Aux Pays-Bas, les fonctionnaires n'ont pas ces avantages (Nations Unies, 2006). Une autre différence marquée, en termes de contrats de travail, est l'usage intensif des contrats temporaires en Espagne, surtout dans le secteur privé. Dans notre fenêtre d'observation, la proportion d'employés dans des contrats a durée déterminée est en moyenne de 30\% en Espagne, un niveau bien plus élevé qu'en Italie (10\%), Allemagne (12\%), en France ou aux Pays-Bas (14\%) (Commission Européenne, 2004).

De même pour le recrutement, les modalités de sélection pour les postes de fonctionnaires se distinguent nettement de celles régissant les autres emplois du secteur public. En France, en Italie et en Espagne, l'accès à ces postes s'effectue par un concours ouvert et tenu annuellement. Ces pays recrutent explicitement pour des carrières de long terme dans le fonctionnariat et les recrues potentielles sont évaluées en fonction de leur aptitude à une telle carrière. En revanche, en Allemagne et aux Pays-Bas, le recrutement se fait sur la base d'un poste précis plutôt que d'une carrière à long terme. De plus, ces pays n'utilisent pas de concours national pour le recrutement des fonctionnaires. Une fois embauchés, les fonctionnaires en Allemagne, Italie, Espagne et France profitent d'une sécurité de l'emploi à vie et ne peuvent être licenciés qu'en cas de faute. Par contraste, aux Pays-Bas, la sécurité de l'emploi des fonctionnaires est la même que celle des autres employés. De plus, les promotions et augmentations de salaires n'y dépendent que du mérite et ne sont pas indexés systématiquement sur l'ancienneté. Malgré cette absence de carrière garantie, la plupart des fonctionnaires aux Pays-Bas demeurent au service de l'Etat tout au long de leurs carrières (Nations Unies, 2006). Dans les autres pays, la progression de salaires est déterminée par l'ancienneté de manière presque automatique.

En ce qui concerne les régimes de retraite, seules la France, l'Allemagne et la GrandeBretagne offrent des conditions avantageuses pour leurs fonctionnaires. Les autres employés du secteur public ne bénéficient pas de conditions plus favorables lorsqu'on les compare à leurs homologues du secteur privé.

Les différences institutionnelles détaillées ici peuvent maintenant être rapprochées de nos résultats en termes de prime "vraie" de long terme dans le secteur public. Aux Pays-Bas, nous estimons que cette prime est négative dans le haut de la distribution, ce qui est cohérent avec une économie où les employés du secteur public ne bénéficient pas de conditions avantageuses relatives à la sécurité de l'emploi et à la progression des salaires. 
En Allemagne, où il n'existe pas de concours d'entrée pour le fonctionnariat, la prime est également négative dans les derniers déciles, sans pourtant l'être de manière significative. En Grande-Bretagne, l'emploi public protège surtout de la volatilité des salaires, mais n'offre qu'une prime de long terme positive mais modeste. En revanche, en France, en Italie et en Espagne où les institutions sont assez similaires, nous estimons des primes "vraies" de l'emploi public qui sont positives en moyenne pour les salaires instantanés et pour les valeurs de long terme en France et en Espagne. En Italie, la prime de long terme n'est positive et significative que dans le haut de la distribution. Les structures salariales rigides du secteur public en Espagne et en Italie peuvent aussi expliquer que les rendements de l'expérience y soient moindres que dans le secteur privé.

\section{Conclusion}

Notre analyse descriptive montre que les différences dynamiques entre les trajectoires salariales dans les secteurs public privé sont importantes. Le débat public sur les écarts de salaires entre secteurs est habituellement centré sur des différences statiques de salaires en coupe, alors que des agents à anticipation rationnelles sont tout aussi soucieux des différences de progression et de volatilité des salaires, ainsi que de la sécurité de l'emploi. L'image de l'inégalité entre secteurs présentée dans les travaux empiriques sur les distributions de salaires instantanés dans les deux secteurs n'est par conséquent qu'une représentation incomplète de l'inégalité entre secteurs du point de vue des agents. Notre contribution est d'évaluer ces différences dynamiques au moyen d'une stratégie empirique qui estime conjointement la sélection des individus dans le chômage et les deux secteurs d'emploi et les trajectoires salariales, puis agrége ces résultats sous la forme de simulations de valeurs actualisées de l'emploi dans chaque secteur et pour chaque individu de notre échantillon. Ceci nous permet de contrôler les effets de sélection entre secteurs et d'estimer une prime "vraie" de l'emploi public en termes de valeur actualisée. Nos résultats portent sur la période 1994-2001 et concernent les pays suivants: Pays-Bas, Allemagne, France, Grande-Bretagne, Espagne et Italie. Partout, nous estimons que les effets de sélection représentent une part importante des primes "apparentes" observées dans les données. D'autre part, les primes "vraies", c'est-à-dire les différences estimées une fois les effets de sélection pris en compte, existent (positives ou négatives) dans toutes les dimensions examinées -salaires instantanés, rendement de l'expérience, volatilité des salaires, sécurité de l'emploi.

Lorsque nous agrégeons ces différences, nous estimons que l'emploi dans le secteur public en France et en Espagne offrent des primes "vraies" de long terme qui sont positives et culminent a 8/9 points de log dans le bas de la distribution des salaires. En revanche, 
I'emploi dans le secteur public aux Pays-Bas s'accompagne d'une "prime" négative de 5 points de log sur le long terme pour les employés dans le haut de la distribution.

Sans être en mesure de prouver une causalité entre cadre institutionnel et primes de long terme dans le secteur public, il est intéressant de comparer les institutions régissant le recrutement et les rémunérations dans les pays de notre échantillon et nos résultats en termes de primes de valeurs actualisées. Les grandes lignes qui se dégagent de notre analyse sont les suivantes. Tout d'abord, les Pays Bas diffèrent des autres pays en cela que le secteur public n'y offre pas des trajectoires de carrière et de salaires presque déterministes et que l'emploi public n'est pas attaché à un niveau de sécurité et de privilège similaire aux autres pays que nous observons. Ensuite, l'Espagne, la France et l'Italie sont assez similaires en regard de leur gestion des ressources humaines dans le secteur public: barrières a l'entrée sous la forme d'examens ou de concours spécifiques, accords collectifs concernant les salaires et les promotions, sécurité de l'emploi public.

Dans notre travail structurel, nous présentons un modèle du marché du travail qui nous permet de montrer les interactions entre secteurs public et privé et le rôle de la concurrence entre secteurs pour les services des travailleurs à la recherche d'emploi, venant du chômage ou de l'emploi dans chaque secteur. Les mouvements des employés au sein de chaque secteur et entre secteurs constituent une composante clé de cette concurrence : les premiers permettent aux employés de gravir l'échelle des salaires au sein d'un secteur et augmentent la valeur actualisée de l'emploi dans ce secteur, tandis que les seconds attisent la compétition entre secteurs et exercent une pression sur les décisions salariales des entreprises privées. L'estimation de ce modèle sur des données britanniques offre un bon ajustement statistique, ce qui nous permet d'évaluer l'impact potentiel de plusieurs choix politiques d'emploi et de salaires dans le secteur public en utilisant des résultats simulés de notre modèle. Les choix politiques que nous comparons ont tous trait au débat actuel sur les différents moyens de réduire la masse salariale du secteur public. Nos résultats montrent que les politiques envisagées de réduction de l'emploi et des salaires dans le secteur public n'ont en fait que très peu d'impact sur le niveau d'emploi global et les salaires du secteur privé. En effet, toute diminution de l'emploi public est absorbée en grande partie par un accroissement de l'emploi privé. Certains choix politiques ont cependant un effet substantiel sur la composition de l'emploi dans les deux secteurs, tel que la baisse des salaires qualifiés dans le secteur public qui donne lieu à un mouvement d'une fraction des travailleurs qualifiés du public vers le privé.

Il est important de noter que nos travaux se cantonnent à la description des différentiels de valeurs actualisées entre secteurs public et privé, et à l'évaluation de politiques de réduction d'emploi et/ou de salaires dans le secteur public en simulant leur impact sur le marché du travail global. Les conclusions que nous pouvons apporter se situent donc en aval du débat sur la mission du secteur public et du choix des activités économiques que la puissance publique juge préférable de garder dans le domaine du secteur public. Nos 
travaux ne répondent pas non plus à la question de l'impact sur le bien-être global, c'est-àdire comprenant des effets de bien-être extérieurs au marché du travail, des choix politiques considérés. Ces questions sont l'objet d'un débat plus large qui est au centre d'une littérature de recherche actuelle et à venir. 


\section{Tables}

\section{Table 1}

Description de l'échantillon

\begin{tabular}{rrrrr} 
& & $\mathrm{N}$ & \multicolumn{1}{c}{$\%$} & \multicolumn{1}{c}{$T$} \\
\hline \hline Allemagne & Total & 3402 & $100.0 \%$ & 6.9 \\
\hline \multirow{2}{*}{ Première observation } & Privé & 2422 & $71.2 \%$ & 6.9 \\
& Public & 614 & $18.0 \%$ & 7.1 \\
& Chômage & 366 & $10.8 \%$ & 6.4 \\
\hline
\end{tabular}

\begin{tabular}{rrrrr} 
Pays-Bas & Total & 2564 & $100.0 \%$ & 6.7 \\
\hline \multirow{3}{*}{ Première observation } & Privé & 1858 & $72.5 \%$ & 6.7 \\
& Public & 566 & $22.0 \%$ & 6.8 \\
& Chômage & 140 & $5.5 \%$ & 6.1 \\
\hline
\end{tabular}

\begin{tabular}{rrrrr} 
France & Total & 2619 & $100.0 \%$ & 6.2 \\
\hline \multirow{3}{*}{ Première observation } & Privé & 1634 & $62.4 \%$ & 6.3 \\
& Public & 660 & $25.2 \%$ & 6.4 \\
& Chômage & 325 & $12.4 \%$ & 5.8 \\
\hline
\end{tabular}

\begin{tabular}{rrrrr} 
Italie & Total & 4567 & $100.0 \%$ & 6.6 \\
\hline \multirow{3}{*}{ Première observation } & Privé & 2664 & $58.3 \%$ & 6.7 \\
& Public & 882 & $19.3 \%$ & 6.7 \\
& Chômage & 1021 & $22.4 \%$ & 6.2 \\
\hline
\end{tabular}

\begin{tabular}{rrrrr} 
Espagne & Total & 3689 & $100.0 \%$ & 6.6 \\
\hline \multirow{3}{*}{ Première observation } & Privé & 2311 & $62.6 \%$ & 6.7 \\
& Public & 527 & $14.3 \%$ & 6.7 \\
& Chômage & 851 & $23.1 \%$ & 6.2 \\
\hline
\end{tabular}

\begin{tabular}{rrrrr} 
Royaume-Uni & Total & 2445 & $100.0 \%$ & 7.0 \\
\hline \multirow{3}{*}{ Première observation } & Privé & 1825 & $74.6 \%$ & 7.0 \\
& Public & 357 & $14.6 \%$ & 7.3 \\
& Chômage & 263 & $10.8 \%$ & 6.8 \\
\hline
\end{tabular}

Table 1: Description de l'échantillon 


\section{Table 2}

Différences brutes entre salaires statiques et dynamiques

\begin{tabular}{|c|c|c|c|c|c|c|}
\hline & \multicolumn{2}{|c|}{ Allemagne } & \multicolumn{2}{|c|}{ Pays-Bas } & \multicolumn{2}{|c|}{ France } \\
\hline & Privé & Public & Privé & Public & Privé & Public \\
\hline \multirow{6}{*}{$\begin{array}{l}\text {... moyenne } \\
\text {.. écart type } \\
\text { ce } 1 \text {-période }\end{array}$} & 8.44 & 8.48 & 8.69 & 8.78 & 9.38 & 9.51 \\
\hline & 0.37 & 0.33 & 0.33 & 0.30 & 0.43 & 0.39 \\
\hline & 0.80 & 0.90 & 0.87 & 0.88 & 0.81 & 0.91 \\
\hline & 5.6 & 3.2 & 1.9 & 1.5 & 4.0 & 2.1 \\
\hline & \multicolumn{2}{|c|}{ Italie } & \multicolumn{2}{|c|}{ Espagne } & \multicolumn{2}{|c|}{ Royaume-Uni } \\
\hline & Privé & Public & Privé & Public & Privé & Public \\
\hline \multirow{3}{*}{$\begin{array}{l}\text {... moyenne } \\
\text {...écart type } \\
\text { iance 1-période }\end{array}$} & 7.91 & 8.01 & 12.22 & 12.49 & 7.43 & 7.53 \\
\hline & 0.30 & 0.29 & 0.43 & 0.40 & 0.42 & 0.38 \\
\hline & 0.71 & 0.89 & 0.74 & 0.88 & 0.81 & 0.87 \\
\hline taux de perte d'emploi 1-période & 3.5 & 1.6 & 6.4 & 3.5 & 3.5 & 2.2 \\
\hline
\end{tabular}

Table 2: Différences brutes entre salaires statiques et dynamiques

Salaires bruts mensuels en logarithmes. La prime de salaire mensuel dans le secteur public en France se lit comme la différence entre 9.51 et 9.38 , soit 13 points de log, donc 14.3\%. Les différences statiques se rapportent aux différences de moyennes et d'écarts types des distributions de salaires en coupe. Les différences dynamiques se rapportent aux dynamiques de salaires et de position dans l'emploi, c'est-à-dire ici à l'autocovariance des salaires et au taux de perte d'emploi. 


\section{Table 3}

\section{Distributions des niveaux de qualifications}

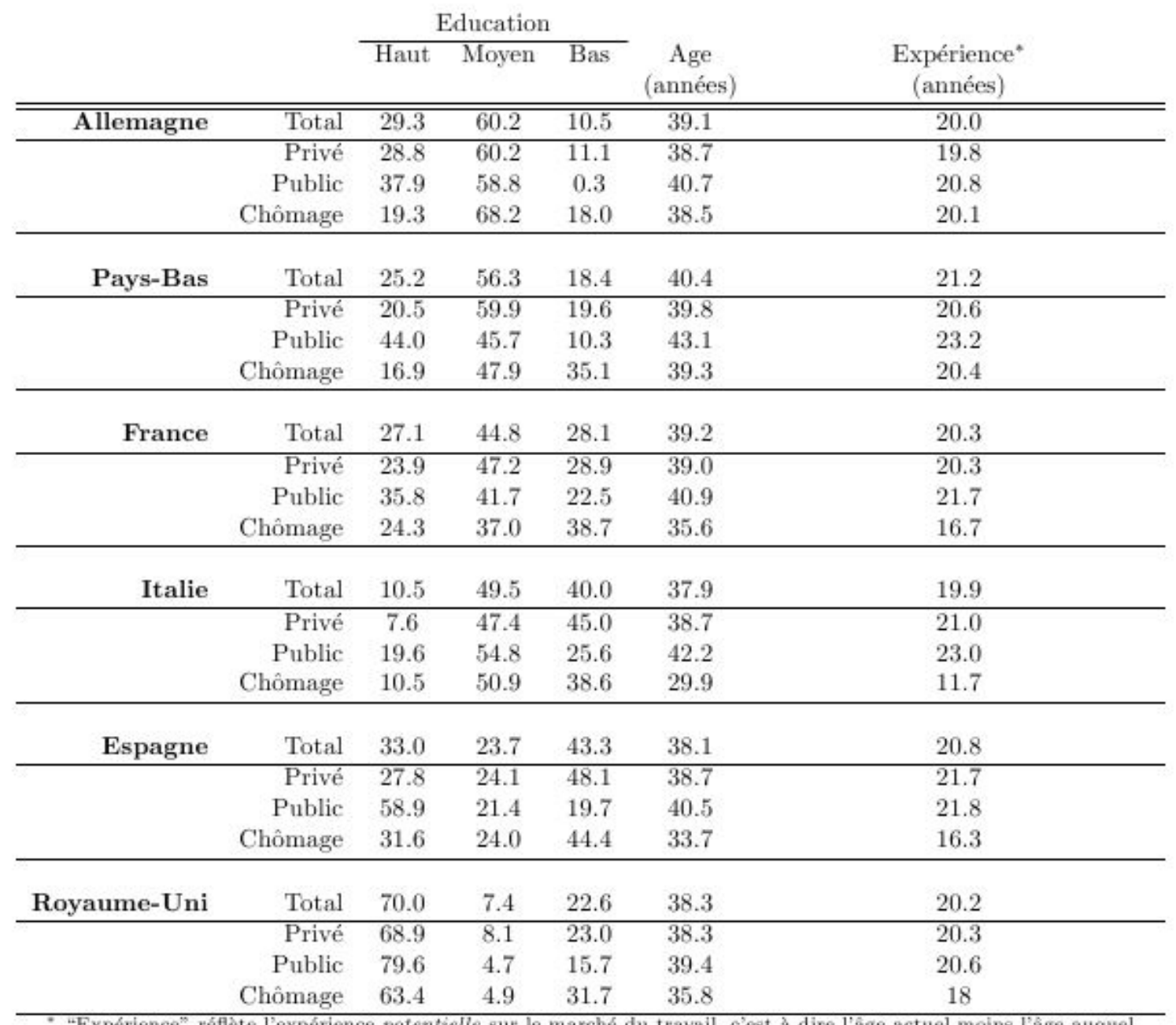

"Expérience" réflète l'expérience potentielle sur le marché du travail, c'est à dire l'âge actuel moins l'âge auquel l'individu est entré pour la première fois sur le marché du travail.

Table 3: Niveaux de qualifications par statut dans l'emploi 


\section{Table 4}

Différences statiques et dynamiques estimées, en contrôlant ou non des effets de sélection

\begin{tabular}{|c|c|c|c|c|c|c|c|}
\hline & & \multicolumn{3}{|c|}{ Différences "vraies" } & \multicolumn{3}{|c|}{ Différences apparentes } \\
\hline \multirow{6}{*}{ Allemagne } & & Privé & Public & Difference & Privé & Public & Difference \\
\hline & moyenne & 8.43 & 8.41 & $\begin{array}{r}-0.02 \\
(0.01)\end{array}$ & 8.43 & 8.47 & $\begin{array}{l}0.04 * \\
(0.01)\end{array}$ \\
\hline & std. dev & 0.32 & 0.29 & $\begin{array}{r}-0.03 * \\
(0.01)\end{array}$ & 0.32 & 0.28 & $\begin{array}{r}-0.04 * \\
(0.01)\end{array}$ \\
\hline & auto-cov & 0.85 & 0.86 & $\begin{array}{l}0.01 \\
(0.02)\end{array}$ & 0.85 & 0.87 & $\begin{array}{l}0.02 \\
(0.02)\end{array}$ \\
\hline & rendements de l'exp. & 0.04 & 0.09 & $\begin{array}{l}0.05 \% \\
\text { (0..01) }\end{array}$ & 0.03 & 0.08 & $\begin{array}{l}0.05 \% \\
0.01\}\end{array}$ \\
\hline & perte d'emploí & 0.08 & 0.10 & $\begin{array}{l}0.03 \S \\
(0.01)\end{array}$ & 0.07 & 0.04 & $\begin{array}{r}-0.03 * \\
(0.01)\end{array}$ \\
\hline \multirow[t]{5}{*}{ Pays-Bas } & moyenne & 8.69 & $8.65^{5}$ & $\begin{array}{r}-0.04 \S \\
(0.02)\end{array}$ & 8.67 & 8.76 & $\begin{array}{l}0.09 * \\
(0.01)\end{array}$ \\
\hline & std. dev & 0.27 & 0.21 & $\begin{array}{c}-0.05 * \\
(0.02)\end{array}$ & 0.27 & 0.22 & $\begin{array}{r}-0.05 * \\
(0.02)\end{array}$ \\
\hline & auto-cov & 0.90 & 0.90 & $\begin{array}{l}0.01 \\
(0.04)\end{array}$ & 0.90 & 0.91 & $\begin{array}{l}0.01 \\
(0.04)\end{array}$ \\
\hline & rendements de l'exp. & 0.11 & 0.13 & $\begin{array}{l}0.01 \\
(0.01)\end{array}$ & 0.12 & 0.10 & $\begin{array}{r}-0.01 \\
(0.01)\end{array}$ \\
\hline & perte d'emploi & 0.03 & 0.01 & $\begin{array}{r}-0.02 \\
(0.04)\end{array}$ & 0.02 & 0.01 & $\begin{array}{l}-0.00 \\
\langle 0.00\rangle\end{array}$ \\
\hline \multirow[t]{5}{*}{ France } & moyenne & 9.37 & 9.40 & $\begin{array}{l}0.03 \S \\
(0.01)\end{array}$ & 9.36 & 9.49 & $\begin{array}{l}0.13 * \\
(0.02)\end{array}$ \\
\hline & std. dev & 0.32 & 0.30 & $\begin{array}{r}-0.02 \\
(0.01)\end{array}$ & 0.32 & 0.31 & $\begin{array}{r}-0.02 \\
(0.01)\end{array}$ \\
\hline & auto-cov & 0.87 & 0.89 & $\begin{array}{l}0.02 \\
(0.03)\end{array}$ & 0.87 & 0.90 & $\begin{array}{l}0.03 \\
(0.02)\end{array}$ \\
\hline & rendements de l'exp. & 0.17 & 0.19 & $\begin{array}{l}0.02 \\
(0.02)\end{array}$ & 0.16 & 0.17 & $\begin{array}{l}0.01 \\
(0.02)\end{array}$ \\
\hline & perte d'emploi & 0.05 & 0.04 & $\begin{array}{r}-0.01 \\
(0.02)\end{array}$ & 0.04 & 0.02 & $\begin{array}{r}-0.02 * \\
(0.00)\end{array}$ \\
\hline \multirow[t]{5}{*}{ Italle } & moyenne & 7.90 & 7.94 & $\begin{array}{l}0.04 * \\
(0.01)\end{array}$ & 7.91 & 8.03 & $\begin{array}{l}0.12 * \\
(0.01)\end{array}$ \\
\hline & std. dev & 0.25 & 0.25 & $\begin{array}{l}0.00 \\
(0.01)\end{array}$ & 0.25 & 0.25 & $\begin{array}{l}0.00 \\
(0.01)\end{array}$ \\
\hline & auto-cov & 0.77 & 0.82 & $\begin{array}{c}0.05 \# \\
(0.03)\end{array}$ & 0.77 & 0.83 & $\begin{array}{l}0.06 \% \\
(0.02)\end{array}$ \\
\hline & rendements de l'exp. & 0.12 & 0.05 & $\begin{array}{c}-0.06 * \\
(0.02)\end{array}$ & 0.10 & 0.04 & $\begin{array}{r}-0.06 \% \\
(0.01)\end{array}$ \\
\hline & perte d'emploi & 0.12 & 0.12 & $\begin{array}{l}0.00 \\
(0.04)\end{array}$ & 0.04 & 0.02 & $\begin{array}{r}-0.02 * \\
(0.01)\end{array}$ \\
\hline \multirow[t]{5}{*}{ Espagne } & moyenne & 12.20 & 12.30 & $\begin{array}{l}0.11 * \\
(0.03)\end{array}$ & 12.21 & 12.47 & $\begin{array}{l}0.26 * \\
(0.02)\end{array}$ \\
\hline & std. dev & 0.36 & 0.30 & $\begin{array}{c}-0.05 \# \\
(0.05)\end{array}$ & 0.35 & 0.32 & $\begin{array}{r}-0.03 \\
(0.02)\end{array}$ \\
\hline & auto-cov & 0.77 & 0.80 & $\begin{array}{l}0.03 \\
(0.10)\end{array}$ & 0.77 & 0.83 & $\begin{array}{l}0.06 \\
(0.07)\end{array}$ \\
\hline & rendements de l'exp. & 0.18 & 0.14 & $\begin{array}{r}-0.04 \\
(0.02)\end{array}$ & 0.16 & 0.13 & $\begin{array}{r}-0.03 \\
(0.02)\end{array}$ \\
\hline & perte d'emploi & 0.12 & 0.10 & $\begin{array}{r}-0.02 \\
(0.03)\end{array}$ & 0.07 & 0.03 & $\begin{array}{r}-0.04 * \\
(0.01)\end{array}$ \\
\hline \multirow[t]{5}{*}{ Royaume-Uni } & moyenne & 7.41 & $7.44 \#$ & $\begin{array}{l}0.03 \\
(0.02)\end{array}$ & 7.42 & 7.50 & $\begin{array}{l}0.09 * \\
(0.02)\end{array}$ \\
\hline & std. dev & 0.38 & 0.34 & $\begin{array}{c}-0.04 \S \\
(0.02)\end{array}$ & 0.39 & 0.35 & $\begin{array}{r}-0.04 \S \\
(0.02)\end{array}$ \\
\hline & auto-cov & 0.83 & 0.88 & $\begin{array}{l}0.05 \\
(0.04)\end{array}$ & 0.83 & 0.88 & $\begin{array}{l}0.06 \\
(0.08)\end{array}$ \\
\hline & rendements de l'exp. & 0.15 & 0.15 & $\begin{array}{c}0.00 \\
(0.02)\end{array}$ & 0.14 & 0.13 & $\begin{array}{r}-0.01 \\
(0.02)\end{array}$ \\
\hline & perte d'emploi & 0.05 & 0.03 & $\begin{array}{r}-0.02 \\
(0.01)\end{array}$ & 0.04 & 0.02 & $\begin{array}{r}-0.01 \S \\
(0.00)\end{array}$ \\
\hline
\end{tabular}

Note: Brreurs-types bootstrap entwe parentbèses. * p<0.01, i p<0.05, \# p<0.10.

Table 4: Différences statiques et dynamiques estimées, en contrôlant ou non des effets de sélection 


\section{Table 5}

\section{Différences de valeurs actualisées et de salaires instantanés}

\begin{tabular}{|c|c|c|c|c|c|c|c|}
\hline & & \multicolumn{3}{|c|}{ Differences -vraies" } & \multicolumn{3}{|c|}{ Différences apparentes } \\
\hline & & 10ème & 50ème & 90ème & 10ème & 50ème & 90ème \\
\hline \multirow[t]{2}{*}{ Allemagne } & Valeur Actualisée & $\begin{array}{l}4.95 \\
(3.08)\end{array}$ & $\begin{array}{l}0.57 \\
(1.98)\end{array}$ & $\begin{array}{r}-2.22 \\
(2.08)\end{array}$ & $\begin{array}{l}8.11 \% \\
(3.09)\end{array}$ & $\begin{array}{l}5.238 \\
(1.88)\end{array}$ & $\begin{array}{l}3.52 \\
(2.17)\end{array}$ \\
\hline & Salaire & $\begin{array}{r}-0.73 \\
(2.27)\end{array}$ & $\begin{array}{r}-3.205 \\
(1.54)\end{array}$ & $\begin{array}{c}-5.33 * \\
(1.76)\end{array}$ & $\begin{array}{l}8.13 \times \\
(2.08)\end{array}$ & $\begin{array}{l}4.458 \\
(1.36)\end{array}$ & $\begin{array}{l}2.85 \\
(1.91)\end{array}$ \\
\hline \multirow[t]{2}{*}{ Pays-Bas } & Valeur Actualisée & $\begin{array}{l}3.54 \\
(3.95)\end{array}$ & $\begin{array}{r}-1.31 \\
\{2.58\}\end{array}$ & $\begin{array}{r}-5.098 \\
(2.23)\end{array}$ & $\begin{array}{l}7.975 \\
(3.56)\end{array}$ & $\begin{array}{l}6.188 \\
(2.14)\end{array}$ & $\begin{array}{l}3.43 \\
(2.26)\end{array}$ \\
\hline & Salaire & $\begin{array}{r}-0.23 \\
(2.98)\end{array}$ & $\begin{array}{r}-2.97 \\
(2.00)\end{array}$ & $\begin{array}{c}-8.69 * \\
(1.99)\end{array}$ & $\underset{(2.22)}{10.99 \mathrm{~s}}$ & $\begin{array}{l}9.80 \times \\
(1.50)\end{array}$ & $\begin{array}{l}5.73 * \\
(2.18)\end{array}$ \\
\hline \multirow[t]{2}{*}{ France } & Valeur Actualiste & $\begin{array}{l}9.058 \\
(3.24)\end{array}$ & $\begin{array}{l}5.698 \\
(1.99)\end{array}$ & $\begin{array}{l}3.10 \\
(2.23)\end{array}$ & $\underset{(3.73)}{13.47 \times}$ & $\frac{12.928}{(2.64)}$ & ${ }_{(2.84)}^{11.22 *}$ \\
\hline & Salaire & $\begin{array}{c}3.96 \# \\
(2.16)\end{array}$ & $\begin{array}{c}2.87 \# \\
(1.69)\end{array}$ & $\begin{array}{l}1.31 \\
(2.21)\end{array}$ & $\underset{(2.23)}{13.20 \%}$ & $\frac{12.60 \%}{(2.04)}$ & $\frac{12.99 *}{(2.94)}$ \\
\hline \multirow[t]{2}{*}{ Italie } & Valeur Actualisée & $\begin{array}{r}-1.87 \\
(1.65)\end{array}$ & $\begin{array}{l}0.25 \\
(1.05)\end{array}$ & $\begin{array}{l}2.29 \\
(1.63)\end{array}$ & $\begin{array}{l}0.48 \\
(2.21)\end{array}$ & $\begin{array}{l}4.21 \# \\
(1.62)\end{array}$ & $\begin{array}{c}15.30 * \\
(2.91)\end{array}$ \\
\hline & Salaire & 7.84s & $\begin{array}{l}3.66 \% \\
(1.01)\end{array}$ & $\begin{array}{l}2.24 \\
(1.65)\end{array}$ & $\underset{(1.25)}{12.50 \%}$ & $\underset{(1.14)}{11.358}$ & ${ }_{(2.23)}^{14.62 *}$ \\
\hline \multirow[t]{2}{*}{ Espagne } & Valeur Actualisíe & $\begin{array}{l}8.455 \\
(3.43)\end{array}$ & $\begin{array}{l}7.17 \# \\
(3.86)\end{array}$ & $\begin{array}{l}4.73 \\
(3.94)\end{array}$ & $\frac{21.74 *}{(3.47)}$ & $\begin{array}{c}26.528 \\
(2.87)\end{array}$ & $\underset{(3.60)}{21.04 *}$ \\
\hline & Salaire & $\begin{array}{c}17.67 \% \\
(4.43)\end{array}$ & $\underset{(3.51)}{10.90 \%}$ & $\begin{array}{l}4.60 \\
(3.99)\end{array}$ & $\underset{(2.92)}{31.44 *}$ & $\underset{(2.26)}{28.428}$ & $\frac{23.80 *}{(2.93)}$ \\
\hline \multirow[t]{2}{*}{ Royaume-Uni } & Valeur Actualisée & $\begin{array}{l}0.53 \\
(4.40)\end{array}$ & $\begin{array}{c}1.06 \\
(2.45)\end{array}$ & $\begin{array}{l}0.52 \\
(2.88)\end{array}$ & $\begin{array}{c}8.35 \# \\
(4.97)\end{array}$ & $\begin{array}{l}3.84 \\
(2.68)\end{array}$ & $\begin{array}{l}2.52 \\
\{2.87\}\end{array}$ \\
\hline & Salaire & $\begin{array}{l}7.075 \\
(3.13)\end{array}$ & $\begin{array}{l}3.955 \\
(2.02)\end{array}$ & $\begin{array}{r}-0.96 \\
(2.48)\end{array}$ & $\begin{array}{c}14.49 \% \\
(3.25)\end{array}$ & $\begin{array}{l}9.51 \% \\
(2.31)\end{array}$ & $\begin{array}{l}3.24 \\
(2.67)\end{array}$ \\
\hline
\end{tabular}

Note: Erreurs-types boatstrap entre parenthèses. ${ }^{*} p<0.01, \mid$ i $p<0.05, \# p<0.10$.

Table 5: Différences de valeurs actualisées et salaires instantanés 


\section{Table 6}

Moyenne et inégalités des salaires et des valeurs actualisées

\begin{tabular}{|c|c|c|c|c|c|c|}
\hline & \multicolumn{3}{|c|}{ Différences -vraies" } & \multicolumn{3}{|c|}{ Différences apparentes } \\
\hline & Privé & Public & Différence & Privé & Public & Différence \\
\hline \multicolumn{7}{|l|}{ Allemagne } \\
\hline Valeur Actualisée moyenne & 11.25 & 11.26 & $\begin{array}{l}0.01 \\
(0.02)\end{array}$ & 11.25 & 11.30 & $\begin{array}{l}0.05 \% \\
(0.02)\end{array}$ \\
\hline Ecart type & 0.36 & 0.33 & $\begin{array}{c}-0.035 \\
\langle 0.01\rangle\end{array}$ & 0.36 & 0.34 & $\begin{array}{r}-0.02 \\
(0.01)\end{array}$ \\
\hline Salaire moyen & 8.40 & 8.37 & $\begin{array}{r}-0.035 \\
\langle 0.02\rangle\end{array}$ & 8.40 & 8.45 & $\begin{array}{l}0.05 \% \\
(0.01)\end{array}$ \\
\hline Ecart type & 0.32 & 0.29 & $\begin{array}{r}-0.03 * \\
(0.01)\end{array}$ & 0.32 & 0.29 & $\begin{array}{r}-0.04 \% \\
(0.01)\end{array}$ \\
\hline \multicolumn{7}{|l|}{ Pays-Bas } \\
\hline Valeur Actualisée moyenne & 11.52 & 11.51 & $\begin{array}{r}-0.01 \\
(0.03)\end{array}$ & 11.50 & 11.56 & $\begin{array}{l}0.065 \\
(0.02)\end{array}$ \\
\hline Ecart type & 0.33 & 0.30 & $\begin{array}{c}-0.04 * \\
(0.01)\end{array}$ & 0.33 & 0.31 & $\begin{array}{r}-0.02 \\
(0.01)\end{array}$ \\
\hline Salaire moyen & 8.67 & 8.63 & $\begin{array}{r}-0.045 \\
\langle 0.02\rangle\end{array}$ & 8.66 & 8.75 & $\begin{array}{l}0.09 \mathrm{x} \\
(0.01)\end{array}$ \\
\hline Ecart type & 0.27 & 0.21 & $\begin{array}{r}-0.05 * \\
(0.02)\end{array}$ & 0.27 & 0.22 & $\begin{array}{r}-0.05 \% \\
(0.02)\end{array}$ \\
\hline \multicolumn{7}{|l|}{ France } \\
\hline Valeur Actualisée moyenne & 12.21 & 12.27 & $\begin{array}{l}0.06 * \\
(0.02)\end{array}$ & 12.19 & 12.31 & $\begin{array}{l}0.13 \% \\
(0.03)\end{array}$ \\
\hline Ecart type & 0.42 & 0.40 & $\begin{array}{r}-0.02 \\
(0.01)\end{array}$ & 0.42 & 0.41 & $\begin{array}{r}-0.01 \\
(0.01)\end{array}$ \\
\hline Salaire moyen & 9.34 & 9.37 & $\begin{array}{c}0.03 \text { \# } \\
(0.02)\end{array}$ & 9.34 & 9.47 & $\begin{array}{l}0.13 \mathrm{x} \\
(0.02)\end{array}$ \\
\hline Ecart type & 0.32 & 0.30 & $\begin{array}{r}-0.02 \\
(0.01)\end{array}$ & 0.32 & 0.30 & $\begin{array}{r}-0.02 \\
(0.01)\end{array}$ \\
\hline \multicolumn{7}{|l|}{ Italie } \\
\hline Valeur Actualisée moyenne & 10.75 & 10.75 & $\begin{array}{l}0.00 \\
(0.01)\end{array}$ & 10.71 & 10.77 & $\begin{array}{l}0.06 \times \\
(0.02)\end{array}$ \\
\hline Ecart type & 0.28 & 0.29 & $\begin{array}{c}0.01 \text { \# } \\
(0.01)\end{array}$ & 0.28 & 0.32 & $\begin{array}{l}0.04 \mathrm{x} \\
(0.01)\end{array}$ \\
\hline Salaire moyen & 7.87 & 7.91 & $\begin{array}{l}0.04 * \\
(0.01)\end{array}$ & 7.90 & 8.02 & $\begin{array}{l}0.12 \times \\
(0.01)\end{array}$ \\
\hline Ecart type & 0.25 & 0.25 & $\begin{array}{l}0.00 \\
(0.01)\end{array}$ & 0.25 & 0.25 & $\begin{array}{l}0.00 \\
(0.01)\end{array}$ \\
\hline \multicolumn{7}{|l|}{ Espagne } \\
\hline Valeur Actualisée moyenne & 15.14 & 15.21 & $\begin{array}{l}0.075 \\
\langle 0.03\rangle\end{array}$ & 15.10 & 15.34 & $\begin{array}{l}0.24 \% \\
(0.03)\end{array}$ \\
\hline Ecart type & 0.38 & 0.37 & $\begin{array}{r}-0.02 \\
(0.02)\end{array}$ & 0.38 & 0.38 & $\begin{array}{r}-0.01 \\
(0.02)\end{array}$ \\
\hline Salaire moyen & 12.15 & 12.26 & $\underset{(0.03)}{0.11 *}$ & 12.18 & 12.46 & $\begin{array}{l}0.28 \mathrm{x} \\
(0.02)\end{array}$ \\
\hline Ecart type & 0.35 & 0.30 & $\begin{array}{r}-0.05 \\
(0.03)\end{array}$ & 0.35 & 0.32 & $\begin{array}{r}-0.02 \\
(0.02)\end{array}$ \\
\hline \multicolumn{7}{|l|}{ Royaume-UnI } \\
\hline Valeur Actualisée moyenne & 10.25 & 10.26 & $\begin{array}{c}0.01 \\
(0.03)\end{array}$ & 10.25 & 10.29 & $\begin{array}{l}0.04 \\
(0.03)\end{array}$ \\
\hline Ecart type & 0.38 & 0.38 & $\begin{array}{r}-0.00 \\
(0.02)\end{array}$ & 0.38 & 0.37 & $\begin{array}{r}-0.02 \\
(0.02)\end{array}$ \\
\hline Salaire moyen & 7.38 & 7.42 & $\begin{array}{c}0.04 \text { \# } \\
(0.02)\end{array}$ & 7.39 & 7.49 & $\begin{array}{l}0.09 \mathrm{~s} \\
(0.02)\end{array}$ \\
\hline Ecart type & 0.38 & 0.34 & $\begin{array}{r}-0.045 \\
\langle 0.02\rangle\end{array}$ & 0.39 & 0.35 & $\begin{array}{r}-0.045 \\
\langle 0.02\rangle\end{array}$ \\
\hline
\end{tabular}

Note: Brreurs-types bootstrap entre parenthèses. ${ }^{+} p<0.01$, i p<0.05, \# $p<0.10$.

Table 6: Moyenne et inegalités des salaires et des valeurs actualisées 


\section{Références}

J. Albrecht, M. Robayo-Abril et S. Vroman [2015] : Public Sector Employment in an Equilibrium Search and Matching Model, mimeo.

Y. Algan, P. Cahuc et A. Zylberberg [2002] : Public Employment and Labor Market Performance, Economic Policy, 17 (34), 7-66.

O. Bargain et B. Melly [2008] : Public Sector Pay Gap in France: New Evidence using Panel Data, IZA Discussion Paper, 3427.

J. Bradley, F. Postel-Vinay et H. Turon, H. [2015] : Public Sector Wage Policy and Labor Market Equilibrium: A Structural Model, Journal of the European Economic Association, à paraitre.

S. Bonhomme et J.-M. Robin [2009] : Assessing the Equalizing Force of Mobility Using Short Panels: France, 1990-2000, Review of Economic Studies, vol. 76(1), pp. 63-92.

A. Bowlus et J. M. Robin [2004] : Twenty Years of Rising Inequality in US Lifetime Labor Income Values, Review of Economic Studies, vol. 71(3), pp. 709-742.

A. Broughton [2009]: Wage Formation in the EU, European Foundation for the Improvement of Living and Working Conditions.

www.eurofound.europa.eu/eiro/studies/tno808019s/tno808019s.htm.

M. Buchinsky et J. Hunt [1999] : Wage Mobility in the United States', Review of Economics and Statistics, vol. 81(3), pp. 351-368.

K. Burdett [2011] : Towards a Theory of the Labor Market with a Public Sector, Labour Economics, vol. 19(1), pp. 68-75.

L. Cappellari [2002] : Earnings Dynamics and Uncertainty in Italy: How Do They Differ between the Private and Public Sectors?, Labour Economics, vol. 9, pp. 477-96.

Commission Européenne [2004] : Fixed-term Contracts in Spain: a Mixed Blessing?, Economic analysis from the European Commission's Directorate-General for Economic and Financial Affairs, vol. 1(1).

Commission Européenne [2013] : Wage Setting Systems and Wage Developments, ec.europa.eu/europe2020/pdf/themes/26l_wageL_settings.pdf 
M. Dickson, F. Postel-Vinay et H. Turon [2014] : The Lifetime Earnings Premium in the Public Sector: The View from Europe, Labour Economics, vol. 31, pp. 141-161.

R. Disney et A. Gosling [2003] : A New Method for Estimating Public Sector Pay Premia: Evidence from Britain in the 1990s, CEPR Discussion Paper, 3787.

P. Du Caju, E. Gautier, D. Momferatou et M. Ward-Warmedinger [2008] : Institutional Features of Wages Bargaining in 23 European Countries, the US and Japan, European Central Bank Working Paper Series, 974.

C. Dustmann et A. van Soest [1998] : Public and Private Sector Wages of Male Workers in Germany, European Economic Review, vol. 42, pp. 1417-1441.

C. Flinn [2002] : Labor Market Structure and Inequality: A Comparison of Italy and the US, Review of Economic Studies, vol. 69(3), pp. 611-645.

P. Ghinetti et C. Lucifora [2007] : Public Sector Pay Gaps and Skill Levels: a Cross-Country Comparison, mimeo.

R. Giordano, D. Depalo, M. Coutinho, B. Pereira, E. Papapetrou, J. Perez, L. Reiss et M. Roter [2011] : The Public Sector Pay Gap in a Selection of Euro Area Countries, European Central Bank Working Paper Series, 1406.

P. Gomes [2015] : Optimal Public Sector Wages, The Economic Journal, vol. 125, pp. 14251451.

P. Gomes [2016] : Heterogeneity and the Public Sector Wage Policy, mimeo.

J. Hartog et H. Oosterbeek [1993] : Public and Private Sector Wages in the Netherlands, European Economic Review, vol. 37, pp. 97-114.

J. Horner, R. Ngai et C. Olivetti [2007] : Public Enterprises And Labor Market Performance, International Economic Review, vol. 48 (2), pp. 363-384.

G. Lassibille [1998] : Wage Gaps Between the Public and Private Sectors in Spain, Economics of Education Review, vol. 17(1), pp. 83-92.

C. Lucifora et D. Meurs [2006] : The Public Sector Pay Gap in France, Great Britain and Italy, Review of Income and Wealth, vol. 52(1), pp. 43-59.

C. Meghir, R. Narita et J.-M. Robin [2015] : Wages and Informality in Developing Countries, The American Economic Review, vol. 105(4), pp. 1509-1546. 
B. Melly [2005] : Public-Private Sector Wage Differentials in Germany: Evidence from Quantile Regression, Empirical Economics, vol. 30, pp. 505-520.

Nations Unies [2006] : Public Administration Country Profiles: Germany, Netherlands, France, Italy, Spain, Division for Public Administration and Development Management (DPADM), Department of Economic and Social Affairs (DESA), http://www.unpan.org/DPADM/ProductsServices/ThematicPortals/PublicAdministration CountryProfiles/newline tabid/677/Default.aspx.

C. Pissarides [2000] : Equilibrium Unemployment Theory, (2nd edition), MIT Press.

F. Postel-Vinay et H. Turon [2007] : The Public Pay Gap in Britain: Small Differences that (don't?) Matter, Economic Journal, vol. 117, pp. 1460-1503.

V. Quadrini et A. Trigari [2007] : Public Employment and the Business Cycle, Scandinavian Journal of Economics, vol. 109 (4), pp. 723-742.

H. van Ophem [1993] : A modified switching regression model for earnings differentials between the public and private sectors in the Netherlands, Review of Economics and Statistics, vol. 75(2), pp. 215-224. 